\title{
How efficient is transport of quantum cargo through multiple highways?
}

\author{
Saptarshi Roy ${ }^{1}$, Tamoghna Das ${ }^{1,2}$, Debmalya Das ${ }^{1,3,4}$, Aditi Sen(De $)^{1}$, Ujjwal Sen ${ }^{1}$ \\ ${ }^{1}$ Harish-Chandra Research Institute, HBNI, Chhatnag Road, Jhunsi, Allahabad 211 019, India. \\ ${ }^{2}$ International Centre for Theory of Quantum Technologies, University of Gdańsk, 80-952 Gdańsk, Poland. \\ ${ }^{3}$ Department of Physics and Astronomy, University of Rochester, Rochester, NY 14627, USA. \\ ${ }^{4}$ Center for Coherence and Quantum Optics, University of Rochester, Rochester, NY 14627, USA.
}

\begin{abstract}
Quantum states can be efficiently transferred over a long distance if the entire quantum channel can be divided into several small blocks. We consider a scenario in which each block consists of two copies of a multiparty state - one is used for distributing an arbitrary quantum state to multiple parties while the other channel is required to concentrate it back to a single party. Both in noiseless and local noisy scenarios, we find one-shot quantum capacities of these channels in terms of fidelity, when the initial shared states in each block are the generalized Greenberger-HorneZeilinger and the generalized $\mathrm{W}$ states. We also consider a situation where optimal local measurements transform multipartite states to bipartite ones which can then be used as single-path channels for quantum state transmission in each segment. We show that in some parameter ranges, the former protocol provides strictly better fidelities than that of the latter, thereby establishing the importance of distributing and concentrating arbitrary quantum states via multipartite entangled states in long distance quantum communication, over the local measurement based protocol. Moreover, we show that in presence of bit flip or bit-phase flip noise, shared generalized Greenberger-Horne-Zeilinger states possess an inherent noise detection and correction mechanism, leading to the same fidelity as in the noiseless case. We consider further noise models also, which do not enjoy the same mechanism. In addition to the fidelity based advantages, the multipath scheme is shown to be useful when one considers a situation in which the completion of the teleportation needs to be delayed. We also find the efficiencies of a quantum channel when a quantum state is transferred over long distances and the entire channel is divided into several small blocks.
\end{abstract}

\section{Introduction}

From the dawn of civilization, communication between different persons and groups played a crucial role in shaping human society. With the emergence of modern science and technologies, a new era in the transmission of information with and without security has been developed. In both the cases, it has been realized, both theoretically $[1,2,4,3,5]$ and experimentally [6, 7], that quantum technologies can provide higher efficiencies [1] than their classical counterparts. In most of the cases, the main ingredient that helps to acheive such advantage is the bipartite entanglement of a shared quantum state between the sender and the receiver [1], (cf. [8, 9, 10, 11, 12, 13] for other quantum information processing tasks).

One of the most interesting consequences of bipartite entanglement is the ability to transfer an arbitrary quantum state, with the additional help of a finite amount of classical communication - quantum teleportation [5]. It is an indispensable feature of quantum mechanics, since to achieve this task by using unentangled states, one requires infinite amount of classical communication between the sender, Alice, and the receiver, Bob (cf. [14, 15]). Significantly, within few years of its discovery, it has been realized experimentally [5, 7], first by using photons [16, 17, 18, 19] and then in other physical substrates [7, 20,21, 22, 23, 24, 25], thereby establishing a new epoch in communication, which can potentially lead to a quantum internet [10], parallel to the extremely useful internet already in use.

One of the avenues by which this breakthrough can become more prominent is by the involvement of multiple parties, which will be a step-forward towards building a quantum network. Over the years, several quantum information transmission protocols with multipartite states were proposed and some of them have also been demonstrated 
in experiments $[27,26]$. On the other hand, if Alice and Bob are located over a long distance, it has been noticed that the task of teleporting an unknown quantum state from Alice to Bob by using a single entangled state is not the best resort. For example, in case of photonic systems with polarization degrees of freedom, photon loss approximately becomes exponential with the length of the channel [17, 19], reducing the quantum capacity of a quantum channel. As a remedy, it was proposed that in a noisy environment, quantum state transfer over a long distance can be divided into several small segments, in which entanglement distillation [28] followed by a modified version of quantum teleportation, known as entanglement swapping [29] are performed to obtain quantum efficiency - the entire process being called a quantum repeater $(\mathrm{QR})[30]$.

In this work, we consider a multipath teleportation protocol where the quantum information is distributed through various pathways before concentrating it back to the desired sender. For some parameter ranges, such a protocol offer better fidelity compared to single path schemes both in the presence and absence of noise. Having specified one advantage of using multiple pathways instead of a single one, let us now mention another advantage of the same, which however preassumes the existence of a quantum memory. In this case, we consider a teleportation protocol in which Alice would like to teleport an arbitrary state to Bob, but at a predetermined later time, at which she would not be able to be present at her port. Therefore, the process has to be mediated by an intermediate party (Claire) who would complete the objective of the process. However, if the intermediate party is not trustworthy, she/he might teleport the state before the predecided time. To reduce this possibility, Alice decides to resort to multiple Claires, so that the process is completed if and only if all the mediators complete their actions. Therefore, even if some Claires break the trust, Bob does not receive the state before the predecided time. More Claires would naturally imply a better assurance in terms of the completion of the protocol in the required time. The above two arguments explain the primary motivation behind considering a multipath teleportation scheme.

At this point, we want to mention that to distribute entanglement over large distance, there are two important schemes in the literature - entanglement percolation [31] and quantum repeaters [30]. In the classical percolation of entanglement (CEP), non maximally entangled or less entangled states are transferred to singlets or Greenberger Horne Zeilinger (GHZ) states with an optimal probability by local operations followed by classical communication and then entanglement swapping leads to the distribution of entanglement among nodes situated in distant location. It was shown that depending on the lattice structure, if one initially performs certain operations which can transform lattice to an optimal setting, and then CEP can be applied, one can enhance the probability of entanglement distribution. On the other hand, for repeaters, starting from many copies of a noisy entangled states, a maximally entangled state is created via distillation and after that, entanglement swapping is performed between adjacent nodes to create entangled states in distant parties.

Inspired by recent experimental achievements which establish an entangled channel over a few thousand kilometers [19] and developments on QRs [32], we consider a quantum state transmission protocol, which is divided into several small blocks, involving mutipartite states. In particular, each block consists of two multipartite states, among which one is used to transfer an arbitrary quantum state among multiple parties while the other one is for accumulating it back to a single one. We call this process as the "multipath quantum repeater" which has two components, "teledistribution" and "teleconcentration" (TD-TC) (see Fig. 1). The port which possesses the initial state to be transferred can be referred as an input port while the receiver who finally receives the unknown state is the output port, and other parties involved in this protocol can be called the auxiliary nodes. To summarize, Alice achieves the delayed teleportation without she being available at her port but also ensuring the specific time of teleportation by:

1. Invoking a third party, Claire, between Alice and Bob who would mediate the teleportation process at the predecided time.

2. Involving two or more Claires which provides a better assurance of the perfect timing of the protocol even if some of the intermediates do not abide by the temporal guideline.

As we will report, the results obtained in this scenario also shows some other advantages of this process. Of course, there is an established fact that sharing entangled state in a smaller distance compared to a longer one, is always advantageous due to the presence of decoherence.

The multiple-path protocol can reduce to a bipartite linear-chain scenario if all the auxiliary parties excepting one, perform optimal local measurements - we refer to such scheme as "local measurement-based single-path QR". Such a situation might be relevant when one of the Claires refuses to take part in the protocol but assists it, in terms of the fidelity yield, to the best of her ability by optimal local measurements. 


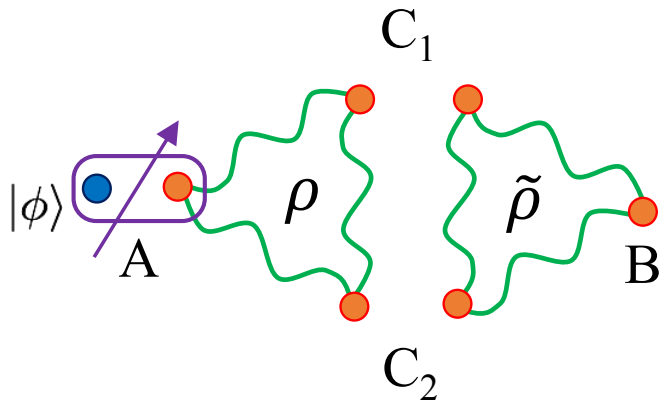

$A$ encodes information

(a)

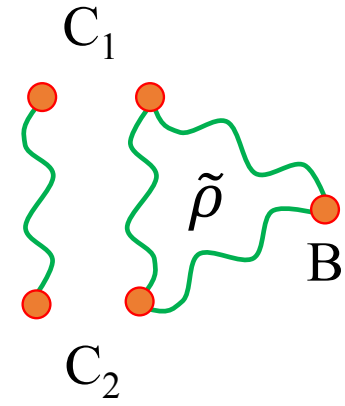

$A$ leaves the protocol

(b)

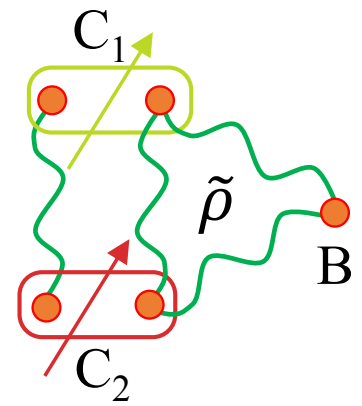

Teleportation completed

(c)

Figure 1: A schematic diagram of multipath quantum repeater. It consists of teledistribution and teleconcentration parts. A sender, Alice, $A$, who posses a unknown quantum state to be teleported to a receiver, Bob, denoted by $B$ shares a multiparty state, $\rho$, with Claires, $C_{1}$ and $C_{2}$, while $B$ is also connected with the same Claires via another multiparty state, $\tilde{\rho}$. $A$ and $B$ are respectively called input and output ports while Claires are the auxiliary nodes. (a)- First step: $A$ performs a joint measurement on her part of the shared state and the state to be teleported. (b)- The scenario when $A$ has left the protocol after the joint measurement by $A$ and unitary operations by $C_{1}$ and $C_{2}$ on $\rho$. (c) Last step is when $C_{1}$ and $C_{2}$ perform their measurements and complete the process.

In this paper, we compare (one shot) quantum capacities, in terms of fidelities, obtained in these two processes and report that both in noiseless and noisy scenarios, TD-TC protocol can yield higher fidelity, than that of the local measurement-based single-path QR, for a set of three-qubit states, justifying the importance of multipartite states, in QR process. Specifically, in a noiseless case, we demonstrate this advantage when the shared state is the three-qubit generalized W ( $\mathrm{gW}$ ) [35] state for certain range of parameters although such benefit is not observed for the family of generalized Greenberger-Horne-Zeillinger (gGHZ) states [36]. Interestingly, in presence of local noise in one or in both the auxiliary ports, the $\mathrm{gGHZ}$ and the $\mathrm{gW}$ states, for some choices of parameters show a clear advantage of TD-TC scheme with multiple paths over the single-path one. Moreover, we find that in the TD-TC protocol, the gGHZ state possesses an inherent local noise detection and rectification mechanism for a specific type of noisy environment. Other relevant studies in similar lines include distribution accompanied by error correction of quantum information [33] and remote information extraction during teledistribution [34]. For a quantitative analysis, a numerical simulation is performed which gives the percentages of shared initial states, having better fidelity in multipath protocol than the local measurement-based ones in presence of several paradigmatic noise models [37, 38], namely, bit flip, phase flip, bit-phase flip, amplitude damping and phase damping channels. By using recursion relation, we finally obtain quantum capacities of a quantum channel over a large distance, having arbitrary number of blocks, when the gGHZ and the $\mathrm{gW}$ states are used in each block, performing multipath as well as single-path schemes. In case of the gGHZ states, we also show that more mediators lead to the same fidelity as obtained with two mediators, even in the presence of bit flip, phase flip, bit phase flip noises.

In our considered protocol, each block is constructed out of single copies of multipartite states enabling a one-shot process. It bears a strong resemblance with quantum repeaters without the initial step of entanglement distillation. We proceed to work with the states at hand without attempting to enhance its entanglement. Specifically, by performing measurements in different nodes (entanglement swapping), we establish entangled connections between two distant nodes. The novelty of our work lies on the fact that the proposal involves entanglement distribution followed by entanglement concentration which have distinct advantages like time delayed teleportation, detection and rectification of certain noise, etc. as mentioned before. Furthermore, compared to the percolation scheme, our method can be applied to obtain fidelities for some mixed states as well, specifically those states that suffer from various local noise models. Note however that the computations of fidelity as well as designing the measurement-based protocol become harder both the noisy case and for initial shared mixed states.

The paper is arranged as follows. Sec. 2 is devoted to a more detailed discussion of TD-TC protocol involving multipartite entangled states, between the source and the receiver. In Sec. 3, we discuss the entire protocol in details 
for two families of three-qubit pure states, namely the generalized GHZ and the generalized W states belonging to two inequivalent SLOCC classes of three-qubit states, as quantum channels in a multipath protocol while optimal fidelities in local measurement-based case are also derived for the similar families of multiparty quantum states in Sec. 4 for comparison. Sec. 5 deals with the case when one of the parties are affected by different kinds of local noise. Before conclusion (Sec. 8), we discuss the capacities of quantum state transfer when the entire distance is divided into several small blocks (see Sec. 6).

\section{Multipath Quantum Repeater: Setting the stage}

In this section, we will discuss a communication protocol where a sender and a receiver are connected via multiple channels. Alice, $A$, intends to send an unknown quantum state, $|\psi\rangle$, given by

$$
|\psi\rangle=a|0\rangle+b|1\rangle
$$

with $a$ and $b$ being complex numbers, satisfying $|a|^{2}+|b|^{2}=1$, to Bob, $B$, with the help of $N-1$ Claires, $C_{1}, C_{2}, \ldots, C_{N-1}$. Two multiparty quantum states, $\rho_{N}$, and $\tilde{\rho}_{N}$ are shared between Alice and Claires, and between Claires and the Bob respectively (See Fig. 1 for illustration with $N=3$ ). The protocol consists of the following steps:

Step 1. Alice initiates the process by performing a joint measurement, $M_{A}$, on the unknown input state $|\psi\rangle$, and her part of the quantum state $\rho_{N}$.

Step 2. Alice communicates her measurement outcome classically to the Claires, $C_{1}, C_{2}, \ldots, C_{N-1}$, post which each of them performs local unitary operations, $\left\{U_{C_{i}}^{j}\right\}, i=1, \ldots, N-1, j=1, \ldots d$, on their respective parts of the shared state $\rho_{N}$, where $d$ is the number of elements in the measurement basis. Alice may now leave the protocol.

Step 3. The $i^{\text {th }}$ Claire performs a measurement $\left\{M_{C_{i}}\right\}$, jointly on her part of the rotated post-measured state of $\rho_{N}$ and her part of the shared $\tilde{\rho}_{N}$, at some later time which is predecided.

Step 4. Based on the measurement results communicated by the Claires, Bob rotates his part of the quantum state with unitary operators, $\left\{U_{B}^{k}\right\}$. We refer to such a protocol as "multipath QR", where Steps 1 and 2 are parts of TD while Steps 3 and 4 together constitutes the TC (for the same, (cf. [39])).

Let us suppose that at the end of the protocol, Bob obtains the state $\rho_{\psi}$. The fidelity under TD -TC is then defined as

$$
\tilde{\mathcal{F}}^{D C}=\int\left\langle\psi\left|\rho_{\psi}\right| \psi\right\rangle d \psi
$$

which depends on the choice of measurements, $M_{A},\left\{M_{C_{i}}\right\}_{i=1}^{N-1}$ at the Alice's and $N-1$ Claires' nodes respectively and also on the unitary rotations $\left\{U_{C_{i}}^{j}\right\}$ and $\left\{U_{B}^{k}\right\}$. Hence, the optimal fidelity of multipath QR by shared multiparty quantum channels, $\rho_{N}$ and $\tilde{\rho}_{N}$, is obtained by maximizing Eq. (2), over all measurement strategies and unitary operators, given by

$$
\mathcal{F}^{D C}\left(\rho_{N}, \tilde{\rho}_{N}\right)=\max _{M_{A},\left\{M_{C_{i}}\right\},\left\{U_{C_{i}}^{j}\right\},\left\{U_{B}^{k}\right\}} \int\left\langle\psi\left|\rho_{\psi}\right| \psi\right\rangle d \psi .
$$

Note that if a sender and a receiver share a quantum state having vanishing entanglement, the teleportation fidelity of sending a qubit can not go beyond $\frac{2}{3}$, with the help of classical communication, while the fidelity reduces to $\frac{1}{2}$, when there is no classical communication allowed between them. In this paper, whenever we encounter a product state, shared between Alice and Claires or between the Claires and Bob, we put the value of fidelity to be $\frac{2}{3}$. Note that a pure quantum state between two parties, if without entanglement, can only be product.

Before presenting the results, let us discuss the scenario where multipath QR can be useful. The advantages of QR is well known and hence we only concentrate on the benefit of TD-TC protocol. Apart from the advantages obtained in terms of fidelity, we discuss the importance of the auxiliary nodes in the protocol and then the benefit of several nodes instead of a single one. First of all, consider a scenario where Alice wants to send an unknown quantum state at some predecided later time, when Alice is not available and Claires are ready to help Alice. In particular, Claires' presence is essential when Alice needs to leave the laboratory at some earlier time, or the location of her laboratory is in such a place that she could not be present at the time of transmission. For example, such a situation can arise if 
Alice is a part of secret agency and she has to perform her measurement as soon as she gets the unknown state, any delay on her part can cause unnecessary threat to her as well as to the protocol. Secondly, it can also be argued that if the protocol involve a single Claires, and if she is "uncooperative" or is compromised by any third party (enemy), there is a possibility of a measurement and a feed forward procedure, resulting in information leakage before the time. We will show that the introduction of many Claires, not only helps Alice-Bob to overcome Alice's constraints, but also leads to a better fidelity compared to a bipartite scenario. In this paper, we also assume that the Claires are not allowed to communicate between each other even classically, and classical communication is only between (Alice, $\left.C_{i}\right)(i=1,2, \ldots N)$ and between $\left(C_{i}\right.$, Bob) pairs.

Our aim here is to analyze the performance of the shared quantum channels, both noiseless and noisy, in terms of fidelity of the TD-TC protocol defined in Eq. (3). However, finding optimal fidelity after maximizing over measurements and unitaries in a multipartite scenario is not easy. As a way-out, looking at the symmetries of the states involved, we choose a particular kind of measurement and unitary operators, which provides a lower bound on $\mathcal{F}(\rho)$. In this paper, we deal with two information-theoretically important families of three-qubit pure states, the generalized Greenberger-Horne-Zeillinger (GHZ) [36], and the generalized W states [35], shared between $\left(A, C_{1}, C_{2}\right)$ and $\left(C_{1}, C_{2}, B\right)$-trios. In the subsequent calculations, we do not explicitly mention about the time delay issue but only consider the fidelity yield of various configurations considered in this manuscript. However, note that all these strategies can be easily continued to fit the conditions of delayed teleportation.

\section{Two-path quantum repeater: Noiseless scenario}

In this section, we assume that the shared three-qubit states used as quantum channels are noiseless, and we explicitly discuss the protocols as well as the evaluation of their fidelities. The effects of local noise on the protocol will be discussed in the succeeding sections.

\subsection{TD-TC protocol via Generalized GHZ state}

Let us first consider the situation, in which Alice shares a three-qubit generalized GHZ state $|g G H Z\rangle$, given by

$$
|g G H Z(\alpha)\rangle=\sqrt{\alpha}|000\rangle+\sqrt{1-\alpha} e^{i \phi}|111\rangle,
$$

where $\alpha \in(0,1)$ and $\phi \in[0,2 \pi)$, with $C_{1}$ and $C_{2}$. The receiver, Bob, shares another copy of the same state, $|g G H Z(\alpha)\rangle$, with the auxiliary nodes, $C_{1}$ and $C_{2}$. Note here that all the parties except Bob (to whom the unknown state has to be teleported) initially possess two qubits. Alice first performs a joint Bell measurement $\left\{\left|\phi^{ \pm}\right\rangle,\left|\psi^{ \pm}\right\rangle\right\}[40]$ on the input state $|\psi\rangle$, and the subsystem of $|g G H Z(\alpha)\rangle$ in her possession. The unnormalized post measured states (PMS) at $C_{1}$ and $C_{2}$ read as

$$
\begin{aligned}
\left|\xi_{\phi}^{ \pm}\right\rangle_{C_{1} C_{2}} & ={ }_{A^{\prime} A}\left\langle\phi ^ { \pm } \left|\left(|\psi\rangle_{A^{\prime}} \otimes|g G H Z\rangle_{A C_{1} C_{2}}\right),\right.\right. \\
& =\left(a \sqrt{\alpha}|00\rangle \pm b \sqrt{1-\alpha} e^{i \phi}|11\rangle\right) / \sqrt{2} \\
\left|\xi_{\psi}^{ \pm}\right\rangle_{C_{1} C_{2}} & ={ }_{A^{\prime} A}\left\langle\psi ^ { \pm } \left|\left(|\psi\rangle_{A^{\prime}} \otimes|g G H Z\rangle_{A C_{1} C_{2}}\right),\right.\right. \\
& =\left(b \sqrt{\alpha}|00\rangle \pm a \sqrt{1-\alpha} e^{i \phi}|11\rangle\right) / \sqrt{2} .
\end{aligned}
$$

Depending on the measurement outcomes obtained and communicated by Alice, the Claires perform local unitary operations, chosen from the Pauli operators, $\left\{I, \sigma_{x}, \sigma_{y}, \sigma_{z}\right\}$ on their respective parts of the shared $|g G H Z(\alpha)\rangle$ state. The set of local unitaries at the end of $C_{1}$ and $C_{2}$ can jointly be represented as $\left\{I \otimes I, I \otimes \sigma_{z}, \sigma_{x} \otimes \sigma_{x}, \sigma_{x} \otimes \sigma_{y}\right\}$, corresponding to $\left\{\left|\phi^{ \pm}\right\rangle,\left|\psi^{ \pm}\right\rangle\right\}$clicks.

After performing these unitary operations, the PMS shared by $C_{1}$ and $C_{2}$ are the $\left|\xi_{\phi}^{+}\right\rangle_{C_{1} C_{2}}$ and $\left|\xi_{\psi}^{+}\right\rangle_{C_{1} C_{2}}$, without normalization. Note that the normalization constants of these states are the probabilities of obtaining the outcomes of the Bell measurement performed by Alice. The first two steps is a part of TD protocol. After this, $C_{1}$ and $C_{2}$ perform two independent Bell measurements on their auxiliary nodes, communicate their measurement results to Bob and finally Bob chooses unitary operators given in Ref. [41], depending on the eight measurement outcomes $\left\{\left|\phi^{ \pm}\right\rangle \otimes\left|\phi^{ \pm}\right\rangle\right\}$ and $\left\{\left|\psi^{ \pm}\right\rangle \otimes\left|\psi^{ \pm}\right\rangle\right\}$. An interesting point to note here is that the structure of the gGHZ state guarantees that if $\left|\phi^{ \pm}\right\rangle$clicks at $C_{1}$ 's port, then $\left|\psi^{ \pm}\right\rangle$can never click at $C_{2}$ 's end and vice-versa. Thus we can have only eight possible outcomes of 


\begin{tabular}{|c|c|c|}
\hline \multicolumn{2}{|c|}{ Outcomes by } & Teleported state to \\
\hline$C_{1}$ & $C_{2}$ & $\operatorname{Bob}(B)$ \\
\hline \multicolumn{3}{|c|}{ When Alice obtains $\left|\phi^{ \pm}\right\rangle$} \\
\hline$\left|\phi^{ \pm}\right\rangle$ & $\left|\phi^{ \pm}\right\rangle$ & $\left(a \alpha|0\rangle+b(1-\alpha) e^{2 i \phi}|1\rangle\right) / 2 \sqrt{2}$ \\
\hline$\left|\psi^{ \pm}\right\rangle$ & $\left|\psi^{ \pm}\right\rangle$ & $\sqrt{\alpha(1-\alpha)} e^{i \phi}(a|0\rangle+b|1\rangle) / 2 \sqrt{2}$ \\
\hline \multicolumn{3}{|c|}{ When Alice obtains $\left|\psi^{ \pm}\right\rangle$} \\
\hline$\left|\phi^{ \pm}\right\rangle$ & $\left|\phi^{ \pm}\right\rangle$ & $\sqrt{\alpha(1-\alpha)} e^{i \phi}(a|0\rangle+b|1\rangle) / 2 \sqrt{2}$ \\
\hline$\left|\psi^{ \pm}\right\rangle$ & $\left|\psi^{ \pm}\right\rangle$ & $\left(a(1-\alpha) e^{2 i \phi}|0\rangle+b \alpha|1\rangle\right) / 2 \sqrt{2}$ \\
\hline
\end{tabular}

Table 1: Table of four possible teleported states at the output port, B, after different measurement outcomes by Alice and Claires, $C_{1}$ and $C_{2}$ and the unitary rotations by Claires and Bob.

the measurements instead of sixteen, which would later turn out to be useful in a noisy scenario. In each of the cases, the (unnormalized) quantum state, teleported to Bob, is given in Table 1.

Therefore, the fidelity in multipath QR by using gGHZ state is constrained by

$$
\begin{aligned}
\mathcal{F}^{D C}(g G H Z) \geq & \int d^{2} a d^{2} b\left(\left.|| a\right|^{2} \alpha+\left.|b|^{2}(1-\alpha) e^{2 i \phi}\right|^{2}\right. \\
& \left.+2 \alpha(1-\alpha)+\left.|| a\right|^{2}(1-\alpha) e^{2 i \phi}+\left.|b|^{2} \alpha\right|^{2}\right), \\
= & \frac{2}{3}+\frac{4}{3} \alpha(1-\alpha) \cos ^{2} \phi .
\end{aligned}
$$

The lower bound on $\mathcal{F}^{D C}(g G H Z)$ can be improved if one absorbs the phase factor of the gGHZ state to any one of the measurements by the Claires or in any one of the unitary operations by Claires or Bob. For example, in the Bell measurement, performed by Alice, she can choose the basis by redefining $|1\rangle \rightarrow|\tilde{1}\rangle=e^{i \phi}|1\rangle$. Immediately, Eq. (7) reduces to

$$
\mathcal{F}^{D C}(g G H Z(\alpha)) \geq \frac{2}{3}+\frac{4}{3} \alpha(1-\alpha) \equiv F(g G H Z) .
$$

Notice that it reaches to unity, when $\alpha=\frac{1}{2}$, implying the protocol to be optimal when the shared multiparty quantum state is the GHZ state. It is tempting to conjecture at this point that the TD-TC protocol described above is possibly the optimal one also for the gGHZ state. Furthermore, we would show in a later section that the fidelity obtained above is independent of the the number of intermediate Claires.

\subsection{Generalized $W$ states as multipath quantum channels}

We now move on to a scenario, where the distribution and concentration channels are from the two-parameter family of three-qubit generalized W states [35], given by

$$
|g W(\alpha, \beta)\rangle=\sqrt{\alpha}|001\rangle+\sqrt{\beta}|010\rangle+\sqrt{1-\alpha-\beta}|100\rangle,
$$

where $\alpha, \beta \in(0,1)$ and $\alpha+\beta<1$. Like the case of the gGHZ state, $\left(A, C_{1}, C_{2}\right)$ and $\left(C_{1}, C_{2}, B\right)$ share two copies of $\mathrm{gW}$ state, $|g W(\alpha, \beta)\rangle$. At the input port and at both the auxiliary nodes, Bell measurements are carried out as before. However, the choice of unitary operators are different than that in the preceding section. $C_{1}$ and $C_{2}$ perform identities if $\left|\phi^{+}\right\rangle$or $\left|\psi^{+}\right\rangle$clicks at Alice's node while for the rest of the measurement outcomes, they operate $\sigma_{z}$ in their subsystems. The applications of these local unitaries reduce the PMS from four to two, shared by $C_{1}$ and $C_{2}$, and are 


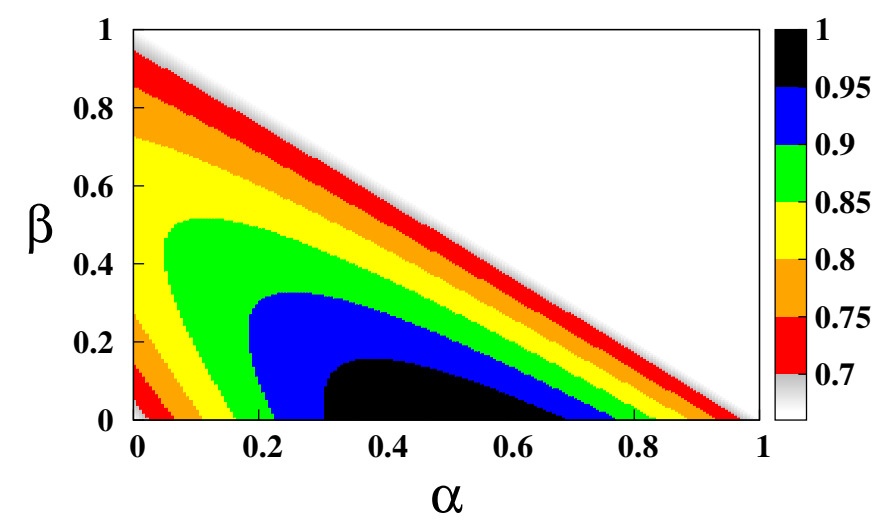

Figure 2: Map of fidelity of a generalized W state vs. state parameters, $\alpha$ and $\beta$, in the multipath QR process. Both axes are dimensionless.

given by

$$
\begin{aligned}
\left|\zeta_{\phi}^{+}\right\rangle_{C_{1} C_{2}} & =\frac{1}{\sqrt{2}}(a(\sqrt{\alpha}|01\rangle+\sqrt{\beta}|10\rangle)+b \sqrt{1-\alpha-\beta}|00\rangle), \\
\left|\zeta_{\psi}^{+}\right\rangle_{C_{1} C_{2}} & =\frac{1}{\sqrt{2}}(a \sqrt{1-\alpha-\beta}|00\rangle+b(\sqrt{\alpha}|01\rangle+\sqrt{\beta}|10\rangle)) .
\end{aligned}
$$

In the TC part, local unitaries on the output port, $B$, are given in Table C.9 of Appendix Appendix C, depending on the results of two Bell measurements, executed by $C_{1}$ and $C_{2}$. Therefore, the fidelity of the TD-TC protocol, described above, for the shared generalized $\mathrm{W}$ states can then be estimated as

$$
\begin{aligned}
\mathcal{F}^{D C}(g W(\alpha, \beta)) \geq F(g W)= & 2 \int d^{2} a d^{2} b\left[\alpha \beta\left(|a|^{4}+|b|^{4}\right)+\frac{1}{4}\left(\left(|a|^{2}(\alpha+\beta)+|b|^{2}(1-\alpha-\beta)\right)^{2}\right.\right. \\
& +\left(|a|^{2}(\alpha-\beta)+|b|^{2}(1-\alpha-\beta)\right)^{2}+\left(|a|^{2}(1-\alpha-\beta)+|b|^{2}(\alpha+\beta)\right)^{2} \\
& \left.\left.+\left(|a|^{2}(1-\alpha-\beta)+|b|^{2}(\alpha-\beta)\right)^{2}\right)\right]+2(\alpha+\beta)(1-\alpha-\beta), \\
= & \frac{2}{3}+\frac{2}{3}(2 \alpha+\beta)(1-\alpha-\beta) .
\end{aligned}
$$

Remark. The behaviour of fidelity for the generalized W state in the $(\alpha, \beta)$-plane is depicted in Fig. 2. From the figure, it is clear that the choice of measurements and unitaries discussed above leads to a maximal fidelity in a region where $\alpha \approx \frac{1}{2}$ and $\beta \approx 0$. This situation arises when the shared $\mathrm{gW}$ state is close to a product state in the $A(B) C_{2}: C_{1}$ bipartitions, having negligible genuine multipartite entanglement. A similar scenario is true when the state is product across $A(B) C_{1}: C_{2}$ bipartition, although the choice of unitary operators in this case needs to be different.

\section{Local measurement-based Single-Path quantum repeater}

Instead of the TD-TC scenario, we now consider a scheme where one of the Claires initially performs optimal local measurements in the shared state, thereby reducing the protocol consisting of two linear quantum channels. As discussed earlier, such a situation is important if one of the Claires decline active participation in the TD-TC scheme 


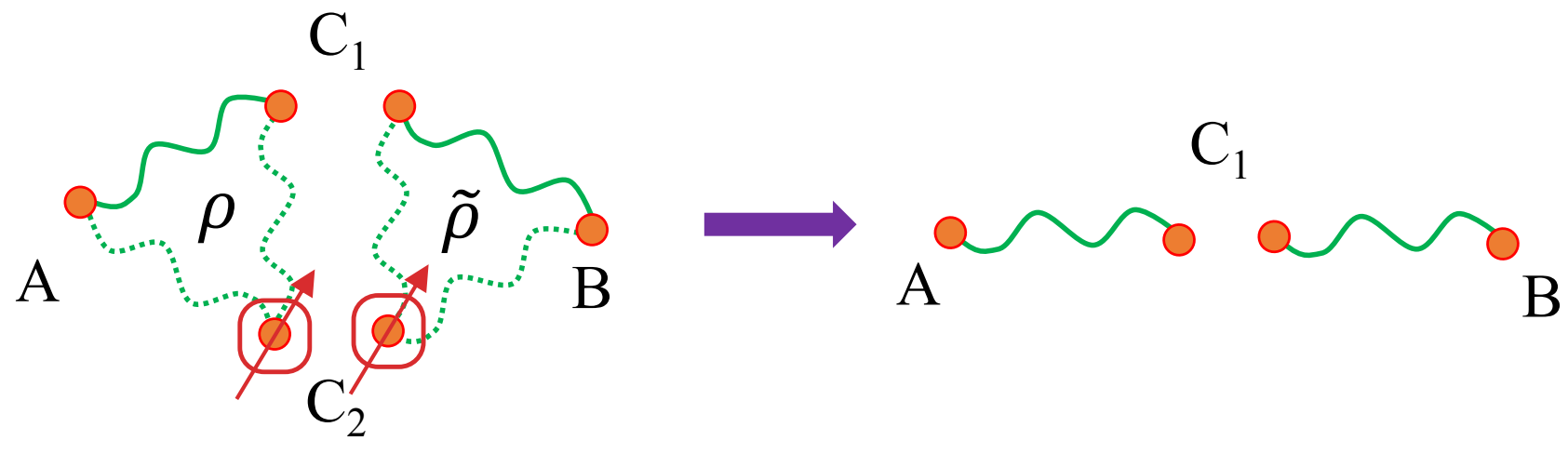

Figure 3: Local measurement-based single-path QR. Optimal local measurements are performed by $C_{2}$, reducing tripartite channels to bipartite ones. For example, executing local measurements by $C_{2}$ disentangles $C_{2}$ from $A$ and $C_{1}$ and the entire TD-TC scheme reduces to the one consisting of $\left(A, C_{1}\right)$ and $\left(C_{1}, B\right)$ duos.

and leaves the protocol before its implementation. Note that this would decrease the guarantee of the perfect timing of the protocol completion, since there is only one Claire left. Nevertheless, our aim here is to compare fidelities obtained by multipath protocol with that of the local measurement-based single-path ones. Specifically, from threeparty quantum states, $\rho$ and $\tilde{\rho}$, shared between $\left(A, C_{1}, C_{2}\right)$ and $\left(C_{1}, C_{2}, B\right)$ trios, certain two-party states, $\sigma_{\rho}$ and $\tilde{\sigma}_{\tilde{\rho}}$ are obtained by operating suitable rank-one projective measurements by one of the Claires, say, $C_{2}$ (see Fig. 3). In this case, $C_{1}$ which actively participates in the protocol can be called functioning port where as $C_{2}$ is the nonfunctioning port. As in the previous case, initially, either two copies of the generalized GHZ or the generalized W state as multiparty channels are shared among all the parties.

The motivation behind considering such a protocol is as follows. First of all, there can be a situation where one of the parties, say $C_{2}$, for some reason, has to leave the protocol, reducing the initial pure state to a mixed state. In case of the gGHZ state, such scenario leads to a separable state between $A$ and $C_{1}$ as well as $C_{1}$ and $B$, although for the $\mathrm{gW}$ state, the reduced state, $P[\sqrt{\beta}|01\rangle+\sqrt{1-\alpha-\beta}|10\rangle]+\alpha P[|00\rangle]$, where $P[|\psi\rangle]=|\psi\rangle\langle\psi|$ is entangled and hence can be used for teleportation protocol with quantum fidelity. The example of the gGHZ state shows that such betrayal or absence can sometimes eliminate the quantum advantage from the communication protocol. Under such circumstances, a profitable situation can be regained if one considers a local measurement-based protocol, if the situation is not of betrayal and the absent party can pre-measure in some basis of his/her parts of the system. In particular, instead of leaving the protocol, $C_{2}$ can help to share highly entangled state to $\left(A, C_{1}\right)$ and $\left(C_{1}, B\right)$ pairs by performing suitable local measurements on her parts, transforming tripartite states to bipartite ones, which are useful for quantum teleportation. In the local measurement-based scenario, after optimization performed over local measurements, the optimal fidelity can be obtained by using the formula of teleportation fidelity in terms of singlet fraction [14]. Note that, similar motivation leads to the concept of entanglement assistance or localizable entanglement [42].

\subsection{Generalized GHZ vs. generalized W states for local measurement-based scheme}

Let us start the discussion with a scenario where an arbitrary three-qubit gGHZ state is shared between the Alice (Bob) and Claires. As mentioned, complete ignorance in the non-functioning port leads to a two party reduced density matrix, given by

$$
\rho_{A(B) C_{1}}^{g G H Z}=\alpha|00\rangle\langle 00|+(1-\alpha)| 11\rangle\langle 11| .
$$

As it is a separable state in the $A(B): C_{1}$ bipartition, $\mathcal{F}_{1}^{l}=\frac{2}{3}$. On the other hand, if $C_{2}$ chooses an arbitrary rank-one projective measurement, given by

$$
\begin{aligned}
|M\rangle & =\sqrt{x}|0\rangle+e^{i \theta} \sqrt{1-x}|1\rangle, \\
\left|M^{\perp}\right\rangle & =\sqrt{1-x}|0\rangle-\sqrt{x} e^{i \theta}|1\rangle,
\end{aligned}
$$




\begin{tabular}{|c|c|c|c|}
\hline Outcome & Probability & Normalised PMS & Schmidt form \\
\hline$|M\rangle$ & $p_{M}$ & $\frac{1}{\sqrt{p_{M}}}\left(e^{-i \phi} \sqrt{\alpha(1-x)}|00\rangle+\sqrt{\beta x}|01\rangle+\sqrt{x(1-\alpha-\beta)}|10\rangle\right)$ & $\sqrt{\Lambda_{M}^{+}}|\tilde{0} \overline{0}\rangle+\sqrt{\Lambda_{M}^{-}}|\tilde{1} \overline{1}\rangle$ \\
\hline$\left|M^{\perp}\right\rangle$ & $p_{M}^{\perp}$ & $\frac{1}{\sqrt{p_{M}^{\perp}}} \sqrt{\alpha x}|00\rangle+e^{i \phi} \sqrt{\beta(1-x)}|01\rangle+$ & $\sqrt{\Lambda_{M^{\perp}}^{+}}|\tilde{0} \overline{0}\rangle+\sqrt{\Lambda_{M^{\perp}}^{-}}|\tilde{1} \overline{1}\rangle$ \\
\hline
\end{tabular}

Table 2: Table of the post measured states (PMS) and the corresponding probabilities, $p_{M}=\alpha+x-2 \alpha x$ and $p_{M}^{\perp}=1-(\alpha+x-2 \alpha x)$. $\Lambda_{M}^{ \pm}$and $\Lambda_{M^{\perp}}^{ \pm}$are the Schmidt numbers [43] for PMS when $M$ and $M^{\perp}$ clicks, and are given by $\Lambda_{M}^{ \pm}=\frac{1}{2}\left(1 \pm \sqrt{1-\frac{4 x^{2} \beta(1-\alpha-\beta)}{(\alpha+x-2 \alpha x)^{2}}}\right)$ and $\Lambda_{M^{\perp}}^{ \pm}=\frac{1}{2}\left(1 \pm \sqrt{1-\frac{4(1-x)^{2} \beta(1-\alpha-\beta)}{(1-(\alpha+x-2 \alpha x))^{2}}}\right)$. The corresponding Schmidt vectors are labeled as $\{|\tilde{0}\rangle,|\tilde{1}\rangle\}$ and $\{|\overline{0}\rangle,|\overline{1}\rangle\}$ for Alice and $C_{1}$ respectively.

the PMS between Alice (Bob) and $C_{1}$ can be represented as

$$
\begin{aligned}
& \left|\xi_{M}\right\rangle=\frac{1}{\sqrt{p_{M}}}\left(\sqrt{x \alpha}|00\rangle+e^{i(\phi-\theta)} \sqrt{(1-x)(1-\alpha)}|11\rangle\right), \\
& \left|\xi_{M}^{\perp}\right\rangle=\frac{1}{\sqrt{p_{M}^{\perp}}}\left(\sqrt{x(1-\alpha)}|00\rangle-e^{i(\phi-\theta)} \sqrt{x(1-\alpha)}|11\rangle\right),
\end{aligned}
$$

where $p_{M}=x \alpha+(1-x)(1-\alpha)$ is the probability of obtaining $|M\rangle$ and $p_{M}^{\perp}=x(1-\alpha)+(1-x) \alpha$ for $\left|M^{\perp}\right\rangle$. The teleportation fidelity by using PMS as channels between Alice to $C_{1}$ is [14]

$$
f_{\max }=\frac{2}{3}+\frac{2}{3} \sqrt{x \alpha(1-x)(1-\alpha)}
$$

which reaches its maximum value for $x=\frac{1}{2}$, representing the optimal measurement basis as $| \pm\rangle=\frac{1}{\sqrt{2}}(|0\rangle \pm|1\rangle)$. We now assume that the teleportation fidelity, by using single-path QR, attains its maximum value when each segment can teleport at its maximum capacity. Starting from the gGHZ state, optimal measurements on both parts of the shared state by $C_{2}$ lead to the bipartite states shared between Alice (Bob) and $C_{1}$, given by

$$
\left|\mu^{ \pm}\right\rangle_{A(B) C_{1}}^{g G H Z}=\sqrt{\alpha}|00\rangle \pm \sqrt{1-\alpha} e^{i \phi}|11\rangle
$$

with probability $\frac{1}{2}$. The fidelity of single-path QR, via quantum channels, $\left(A, C_{1}\right)$ and $\left(C_{1}, B\right)$ pairs, finally reads

$$
\mathcal{F}_{2}^{l}(g G H Z)=\frac{2}{3}+\frac{4}{3} \alpha(1-\alpha) .
$$

For the details, see Appendix A.

Comparing Eqs. (8) and (21), we find that the fidelities obtained by the specific TD-TC protocol with that of the local measurement-based ones exactly match and therefore, we have

$$
\mathcal{F}^{D C}(g G H Z) \geq \mathcal{F}_{2}^{l}(g G H Z)=F(g G H Z),
$$

where the equality holds for the GHZ state with $\alpha=\frac{1}{2}$. If we now assume that with the shared gGHZ state, the protocol presented in Sec 3 is the optimal one, we have that multipath QR in terms of teleportation fidelity does not provide any advantage over local measurement-based single-path QR. We will show that when the shared state is the $\mathrm{gW}$ state, two scenarios no more remains the same.

Let us now move to the protocol with the initial state being the generalized W state. Let us first state the following proposition.

Proposition: Consider two copies of a generalized $W$ state shared between $A, C_{1}, C_{2}$ and $C_{1}, C_{2}, B$. Suppose that $C_{2}$ 
must remain non-functional during the actual implementation of the teleportation, but agrees to make measurements before. The optimal fidelity in the single-path quantum repeater can be obtained when the local measurements at $C_{2}$ are performed in the computational basis.

Proof: After performing measurements by $C_{2}$ in an arbitrary basis given in Eqs. (15) and (16), the PMS and their probabilities of occurrence can be computed (see Table 2). The maximum teleportation fidelity, $f_{\text {max }}^{g W}$, via PMS from A to $C_{1}$ in terms of the maximal singlet fraction [14], $F_{\text {max }}$, is given by

$$
f_{\max }^{g W}=p_{M} f_{\max }^{M}+p_{M}^{\perp} f_{\text {max }}^{M^{\perp}}
$$

where

$$
\begin{aligned}
f_{\max }^{M\left(M^{\perp}\right)} & =\frac{2 F_{\max }^{M\left(M^{\perp}\right)}+1}{3}, \text { and } \\
F_{\max }^{M\left(M^{\perp}\right)} & =\frac{1}{2}\left(1+2 \sqrt{\Lambda_{M\left(M^{\perp}\right)}^{+} \Lambda_{M\left(M^{\perp}\right)}^{-}}\right) .
\end{aligned}
$$

Substituting values from Table. 2, and maximizing over the measurement basis, we obtain

$$
f_{\max }^{g W}=\frac{2}{3}+\frac{2}{3} \sqrt{\beta(1-\alpha-\beta)}
$$

We now note that if $C_{2}$ makes the measurement in the $\{|0\rangle,|1\rangle\}$ basis, the PMS reduces to

$$
\begin{aligned}
& \left|\zeta_{0}\right\rangle=\frac{1}{\sqrt{p_{0}}}(\sqrt{\beta}|01\rangle+\sqrt{1-\alpha-\beta}|10\rangle), \\
& \left|\zeta_{1}\right\rangle=|00\rangle .
\end{aligned}
$$

with $p_{0}=1-\alpha$ and $p_{1}=\alpha$. Following the protocol described in Sec. 3.2, we can easily find that $f_{\max }^{g W}$ can be maximized. If we now assume that the fidelity of local measurement-based single-path QR is maximized when the fidelities at each segment (i.e., $A \rightarrow C_{1}$ and $C_{1} \rightarrow B$ ) are maximized, the optimal measurement basis is the computational basis.

After proving the optimality of the measurement basis, let us now explicitly evaluate the optimal fidelity in this process. If $C_{2}$ performs measurements in the computational basis on her parts of the shared states, the PMS between Alice (Bob) and $C_{1}$ are given in Table 3. From this table, it is clear that except the first outcome, a pure product state is

\begin{tabular}{|c|c|c|}
\hline Outcome & Probability & Output state \\
\hline$|0\rangle|0\rangle$ & $p_{0} p_{0}$ & $\left|\zeta_{0}\right\rangle_{A C_{1}}\left|\zeta_{0}\right\rangle_{B C_{1}}$ \\
\hline$|0\rangle|1\rangle$ & $p_{0} p_{1}$ & $\left|\zeta_{0}\right\rangle_{A C_{1}}\left|\zeta_{1}\right\rangle_{B C_{1}}$ \\
\hline$|1\rangle|0\rangle$ & $p_{1} p_{0}$ & $\left|\zeta_{1}\right\rangle_{A C_{1}}\left|\zeta_{0}\right\rangle_{B C_{1}}$ \\
\hline$|1\rangle|1\rangle$ & $p_{1} p_{1}$ & $\left|\zeta_{1}\right\rangle_{A C_{1}}\left|\zeta_{1}\right\rangle_{B C_{1}}$ \\
\hline
\end{tabular}

Table 3: Table of measurement outcomes by $C_{2}$. The probabilities of getting the outcomes and the post measured states, shared between Alice (Bob) and $C_{1}$, when two copies of three-qubit generalized W states are shared. The output states, $\left|\zeta_{0}\right\rangle$ and $\left|\zeta_{1}\right\rangle$, are given in Eqs. (27) and (28) respectively.

shared between $A$ and $C_{1}$, or between $C_{1}$ and $B$, or between both in all the three cases, and hence for these situations, the fidelity reduces to $\frac{2}{3}$. In the first situation, i.e., when the outcome is $|0\rangle|0\rangle$, the fidelity reads as $\frac{2}{3}+\frac{4}{3\left(p_{0}\right)^{2}} \beta(1-\alpha-\beta)$. For a detailed calculation see Appendix A. Therefore, the optimal fidelity in the local measurement-based single-path $\mathrm{QR}$, when the initial shared state is the generalized W state, can be shown to be

$$
\begin{aligned}
\mathcal{F}_{2}^{l}(g W) & =\left(1-\left(p_{0}\right)^{2}\right) \frac{2}{3}+\left(p_{0}\right)^{2}\left(\frac{2}{3}+\frac{4}{3\left(p_{0}\right)^{2}} \beta(1-\alpha-\beta)\right) \\
& =\frac{2}{3}+\frac{4}{3} \beta(1-\alpha-\beta) .
\end{aligned}
$$




\begin{tabular}{|c|c|c|}
\hline & \multicolumn{2}{|c|}{ Fidelity } \\
\hline Noise type & Multipath q. repeater & Single path q. repeater \\
\hline Bit flip & $\frac{2}{3}+\frac{4}{3} \alpha(1-\alpha)$ & $\frac{2}{3}\{1-p(1-p)\}+\frac{4}{3} \alpha(1-\alpha)\{1-2 p(1-p)\}$ \\
\hline Phase flip & $\frac{2}{3}+\frac{4}{3}(1-2 p)^{2} \alpha(1-\alpha)$ & $\frac{2}{3}+\frac{4}{3}(1-2 p)^{2} \alpha(1-\alpha)$ \\
\hline Bit phase flip & $\frac{2}{3}+\frac{4}{3}(1-2 p)^{2} \alpha(1-\alpha)$ & $\frac{2}{3}+\frac{4}{3}(1-2 p)^{2} \alpha(1-\alpha)$ \\
\hline Amplitude damping & $\frac{2}{3}+\frac{4}{3} \alpha(1-\alpha)-\frac{2 p}{3}\left(1+\alpha-2 \alpha^{2}-p+2 \alpha p-\alpha^{2} p\right)$ & $\frac{2}{3}+\frac{4(1-p)}{3} \alpha(1-\alpha)$ \\
\hline Phase damping & $\frac{2}{3}+\frac{4}{3} \alpha(1-\alpha)-\frac{4 \alpha p}{3}(2-2 \alpha-p+\alpha p)$ & $\frac{2}{3}+\frac{4}{3} \alpha(1-\alpha)-\frac{4 \alpha p}{3}(2-2 \alpha-p+\alpha p)$ \\
\hline
\end{tabular}

Table 4: Effects of five kinds of paradigmatic noise model on fidelities obtained in the TD-TC and local measurement-based schemes when two copies of the three-qubit gGHZ state are shared between input, auxiliary and output nodes.

Remark. For the shared $\mathrm{gW}$ state, instead of performing any measurement, if $C_{2}$ leaves her laboratory, or if $C_{1}$ tries to communicate the quantum state secretly to Bob ignoring $C_{2}$, one can show that sending unknown quantum state with fidelity better than the classical is still possible, and the fidelity is given by

$$
\mathcal{F}_{\text {mixed }}^{l}(g W)=\frac{2}{3}+\frac{2}{3}(2 \beta(1-\alpha-\beta)-\alpha(1-\alpha)) .
$$

Since, $\alpha(1-\alpha)>0$, we conclude that the local measurement-based protocol is always better than the scheme where $C_{2}$ just leaves the protocol without performing the measurement.

Let us now state one of the main results of the paper. In particular, we compare the quantum capacities of multipath QR with that of the local measurement-based single-path ones. Before discussing the results for the entire family of the $\mathrm{gW}$ state, let us state the following theorem which is true for the $\mathrm{W}$ state with $\alpha=\beta=\frac{1}{3}$ :

Theorem: If two copies of the three-qubit $W$ state are shared between $A, C_{1}, C_{2}$ and $C_{1}, C_{2}$, B, sending an unknown qubit in the teledistribution and teleconcentration protocol is always beneficial than using a single-path quantum repeater, consisting of two bipartite quantum states derived from optimal local measurements in the non-functioning port, and the corresponding advantage is $9.1 \%$ or better.

Proof: For the W state, $|W\rangle=\frac{1}{\sqrt{3}}(|001\rangle+|010\rangle+|100\rangle), \mathcal{F}^{D C}(W) \geq \frac{8}{9}>\frac{22}{27}=\mathcal{F}_{2}^{l}(W)$, by using Eqs. (13) and (30), thereby establishing the advantage of mutipath protocol for sending quantum information over the single-path ones.

The above Theorem holds even for the shared generalized W states, for some values of $\alpha$ and $\beta$. In particular, we show that the TD-TC scheme is better than the optimal local measurement-based ones when

$$
\alpha \geq \frac{\beta}{2}
$$

In general, for $\mathrm{gW}$ states with $\alpha \geq \frac{\beta}{2}$, the TD-TC protocol performs better than the local measurement based scheme by $\frac{(2 \alpha-\beta)(1-\alpha-\beta)}{1+2 \beta(1-\alpha-\beta)} \times 100 \%$ or better. Note, however, that it is still possible that the region in which the TD-TC protocol can not give better fidelity, may show the benefit over the local measurement-based scheme if one can construct optimal measurements and unitaries involved in the two-path protocol.

Note that in the case of local measurement-based single-path quantum repeater, using Bell measurements, we obtain the maximal possible fidelity attainable for non maximally entangled bipartite states. However, restricting to Bell measurements in the multipath setting is an assumption in our case. We are prompted to make this assumption due to two main reasons:

1. Apart from the intrinsic advantage that the multipath protocol offers in terms of security in delaying the teleportation protocol appropriately, we want to compare single-path and multiple path protocols when both of 


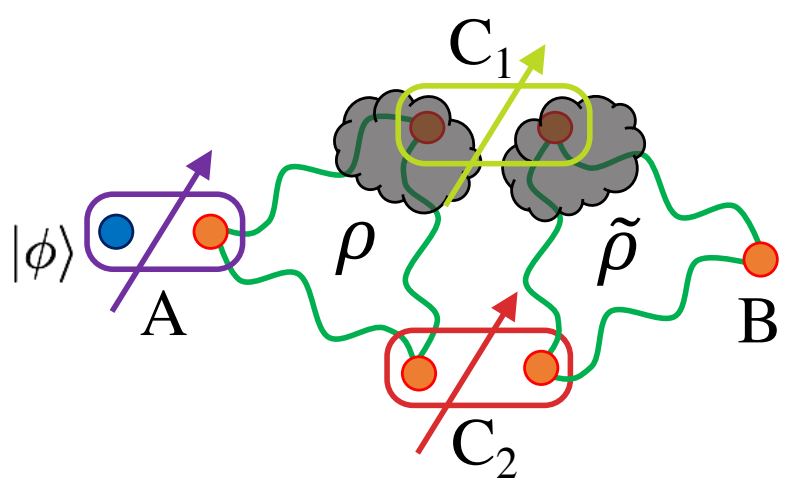

Figure 4: Two-path QR where two qubits of $C_{1}$ are affected by local noises. In case of local measurement-based single-path scheme, local measurements are performed on both the qubits of $C_{2}$ which are not affected by any kind of noise.

them use the same setup. In this situation, we find wide parameter ranges in both noiseless and noisy scenarios (which we deal in the subsequent section), where the later performed better in terms of fidelity and detection as well as correction of local errors. Since the single- path protocol is the optimal one, after optimization over measurements, the fidelity of the multipath scheme can only improve and hence our conclusion remains same.

2. If we want to use any other measurement setting, one requires to perform a nonlinear optimization over multiple parameters which in some sense intractable even numerically.

\section{Noisy channels}

Until now, the results obtained involve quantum channels which are not affected by any kind of noise. In practice, quantum states can never be kept completely isolated from the environment, and hence it is important to investigate the effects of environmental interaction on the quantum capacities of the protocol. In particular, we assume that local noise acts on both the qubits, possessed by $C_{1}$, as shown in Fig. 4 in both multipath and local measurement-based single-path protocols. In this section, we find out the robustness of fidelities, against noise in one of the subsystems of the shared channels. The initial shared state is again two copies of a three-qubit generalized GHZ or a generalized W state. We will consider five different kinds of noise [37] models - (1) Bit flip noise, (2) phase flip noise, (3) bit-phase flip noise, (4) amplitude damping, and (5) phase damping. The detailed actions of these noise models on the quantum state are given in Appendix Appendix B. We assume that irrespective of the noise acting on the subsystems, Alice, Claires $\left(C_{1}\right.$, and $\left.C_{2}\right)$ and Bob continue with their protocol of the noiseless scenario, described in Secs. 2 and 4 . This is probably a natural and important assumption since we believe that the senders and the receivers may not always be in a position to alter their actions, depending on the noise or they may not always be aware of the types of noise acting on the system.

\subsection{Generalized GHZ state against noise: Inherent detection and rectification}

As before, $A(B), C_{1}$ and $C_{2}$ share a $|g G H Z(\alpha)\rangle$ state, and local noise acts on both the subsystems of $C_{1}$. Before comparing the fidelities obtained from the two-path and local measurement-based single-path QRs, let us first discuss that the multipath TD-TC protocol enables us to identify and rectify certain kinds of noise in the system when the $\mathrm{gGHZ}$ state is shared between them.

First note that in the TD-TC scheme, due to the symmetry of the gGHZ states, when $\left|\phi^{ \pm}\right\rangle\left(\left|\psi^{ \pm}\right\rangle\right)$clicks in the measurement performed by $C_{1}$, the outcome of the measurement at the node of $C_{2}$ can not be $\left|\psi^{ \pm}\right\rangle\left(\left|\phi^{ \pm}\right\rangle\right)$in a noiseless scenario or when phase flip error occurs. However, when bit flip or bit-phase flip noise happens on both the qubits of $C_{1}$, such correlations in measurement results are broken and new correlations appear, leading to identification of noise models acting on $C_{1}$. In particular, in these cases, when $\left|\phi^{ \pm}\right\rangle$clicks in $C_{1}$ 's port, $\left|\psi^{ \pm}\right\rangle$can only be the outcome at $C_{2}$ and vice-versa. Therefore, this contrasting feature in the measurement outcomes enables us to conclusively distinguish the local bit flip and bit-phase flip noises acting on one of the Claires' subsystem in the TD-TC protocol 


\begin{tabular}{|c|c|c|}
\hline Noise & \multicolumn{2}{|c|}{ Fidelity } \\
\hline type & Multipath QR & Single-path QR \\
\hline Bit flip & $\begin{array}{c}\frac{2}{3}+\frac{2}{3}((2 \alpha+\beta)(1-\alpha-\beta) \times \\
\left.\left(1-2 p+2 p^{2}\right)+p(p-1)\right)\end{array}$ & $\begin{array}{c}\frac{2}{3}+\frac{4}{3} \beta(1-\alpha-\beta)-\frac{2}{3} p(1-p) \times \\
\left.(1-\alpha)^{2}-4 \beta(1-\alpha-\beta)\right)\end{array}$ \\
\hline \multirow{2}{*}{ Phase flip } & $\frac{2}{3}+\frac{2}{3}\left(2 \alpha(1-\alpha)+\beta(1-\beta)(1-2 p)^{2}\right.$ & $\frac{2}{3}+\frac{4}{3} \beta(1-\alpha-\beta)(1-4 p(1-p))$ \\
\hline Bit phase flip & $\begin{array}{c}3 \alpha \beta+4 \alpha \beta p(1-p)) \\
\left.\left(1-2 p+2 p^{2}\right)+p(p-1)\right)\end{array}$ & $\begin{array}{c}\frac{2}{3}+\frac{4}{3} \beta(1-\alpha-\beta)-\frac{2}{3} p(1-p) \times \\
\left.(1-\alpha)^{2}-4 \beta(1-\alpha-\beta)\right)\end{array}$ \\
\hline Amplitude damping & $\begin{array}{c}\frac{2}{3}+\frac{2}{3}((2 \alpha+\beta)(1-\alpha-\beta) \\
\left.+p\left(\alpha \beta-2 \beta+\beta^{2}(1+p)\right)\right)\end{array}$ & $\begin{array}{c}\frac{2}{3}+\frac{4}{3} \beta(1-\alpha-\beta)-2 \beta p(1-\alpha) \\
+\frac{2}{3} p \beta^{2}(2+p)\end{array}$ \\
\hline Phase damping & $\frac{2}{3}+\frac{2}{3}\left(2 \alpha(1-\alpha)+\beta(1-\beta)(1-p)^{2}\right.$ & $\frac{2}{3}+\frac{4}{3} \beta(1-\alpha-\beta)(1-p(2-p))$ \\
\hline
\end{tabular}

Table 5: Similar consideration as in Table 4 when the shared state is the generalized W state instead of the gGHZ state.

from the noiseless and phase flip noisy scenarios. Interestingly, by designing suitable local unitary operators at Bob's port, the effects of noise, either bit or bit-phase flip errors, on fidelities can be corrected. Let us illustrate the unitary operators of $B$ which are appropriate to correct the bit flip error at the node of $C_{1}$. When the measurement outcomes at the end of $C_{1}$ and $C_{2}$ are $\left\{\left|\phi^{+}\right\rangle\left|\psi^{+}\right\rangle,\left|\phi^{-}\right\rangle\left|\psi^{-}\right\rangle\right\},\left\{\left|\phi^{+}\right\rangle\left|\psi^{-}\right\rangle,\left|\phi^{-}\right\rangle\left|\psi^{+}\right\rangle\right\},\left\{\left|\psi^{+}\right\rangle\left|\phi^{+}\right\rangle,\left|\psi^{-}\right\rangle\left|\phi^{-}\right\rangle\right\}$and $\left\{\left|\psi^{+}\right\rangle\left|\phi^{-}\right\rangle,\left|\psi^{-}\right\rangle\left|\phi^{+}\right\rangle\right\}$, the corresponding unitary operators at the output port can be set to $\left\{I, \sigma_{z}, \sigma_{x}, \sigma_{y}\right\}$ respectively. If Bob now employs the above set of unitaries, the lower bound on the fidelity for TD-TC under bit flip channel coincides with the noiseless case. Interestingly, the the detection and subsequent rectification are not possible in the local measurement-based case, where the fidelity depends on the noise parameter, $p$, (see Table 4). This rectification procedure again shows another superior characteristic of multipath QR protocol over the single-path one.

Suppose now that the sender and the receiver a priori know the more probable error at the $C_{1}$ 's port to be the bit-phase flip one. Hence the circuit for implementing the multipath protocol can be designed in such a way that the fidelity remains unaltered in this scenario. Specifically, the bit-phase flip error can be corrected if Bob applies $\left\{\sigma_{z} \otimes U_{i}\right\}(i=1,2, \ldots, 8)$ where $\left\{U_{i}\right\}$ are the set of unitaries used in the bit flip case. In presence of different kinds of noise models, the lower bounds on $\mathcal{F}^{D C}$, and the exact values of $\mathcal{F}_{2}^{l}$ with the measurement being performed in the $\{|+\rangle,|-\rangle\}$ basis are given in Table 4 for the shared gGHZ state. As discussed before, for bit flip/bit-phase flip errors, the multipath setting turns out to be maximally robust compared to any other noise models while the opposite occurs for the amplitude damping ones, provided the set of measurements and unitaries discussed before in the TD-TC protocol for the shared gGHZ state is optimal. On the other hand, the lower bounds on the TD-TC, match with $\mathcal{F}_{2}^{l}$, when other noises like phase flip, bit-phase flip, phase damping act locally on $C_{1}$, if we assume that the rectifying unitary operators are only applied for bit flip errors.

Remark. For an arbitrary noise model, for example for amplitude damping, when Claires perform measurements in the Bell basis, all possible (sixteen) outcomes can arise, and hence Bob applies all the sixteen unitary rotations mentioned in the noiseless and bit flip cases. In this sense, when the shared state is the gGHZ state, one can always distinguish amplitude damping or other noise models from the errors like bit, phase, bit-phase flips and phase damping channels, although rectification might not always be possible.

\section{Effects of local bit-flip noise on the intermediate ports}

In the above considered situation, we have taken the noise to act on only one node $\left(C_{1}\right)$. Since these two nodes $\left(C_{1}\right.$ and $C_{2}$ ) are at distant (different) locations, we can safely assume that presence or absence of noise in one of the nodes is completely uncorrelated to that of the other. Thus, we argue that it is quite likely that one of the two nodes suffer from local noise, thereby rendering one node to be noisy and the other to be completely error-free. Let us consider 
how the situations when both $C_{1}$ and $C_{2}$ are affected by local noise. Specifically, we present a detailed analysis of fidelity in the presence of bit-flip noise in both the nodes for the gGHZ state. The calculation can be prototypically carried out for other noise models also.

When the shared gGHZ states in both the distribution and concentration sector are affected by noise in both the connecting nodes, the fidelity of the multipath teleportation protocol (designed to correct local bit flip noise in $C_{1}$ ) reads as

$$
\mathcal{F}^{D C}=\frac{2}{3}\{1-q(1-q)\}+\frac{4}{3} \alpha(1-\alpha)\{1-2 q(1-q)\}
$$

Here, $p$ and $q$ are the probabilities with which bit-flip noise acts on $C_{1}$ and $C_{2}$ nodes respectively and the noise on $C_{\text {! }}$ can be corrected as discussed before. Note, when $q=1$ or 0 , the expression of fidelity reduces to that of the noiseless scenario. For intermediate values of $q$, the perfect detection and correction of error is not possible. Nevertheless, in the multiparty scenario, we still obtain an error detection probability of $2 q(1-q)\{1-2 p(1-p)\}+2 p(1-p)\{1-2 q(1-q)\}$. As expected, for $q=1$, the detection probability is unity for all values of $p$, while for $q=1 / 2$, the same falls to $1 / 2$ for the entire $p$-range. The corresponding linear chain fidelity, where $C_{2}$ leaves the protocol after performing the measurement, is given by

$$
\mathcal{F}_{C_{2}}^{L C}=\frac{2}{3}\{1-p(1-p)\}+\frac{4}{3} \alpha(1-\alpha)\{1-2 p(1-p)\}
$$

On the other hand, when $C_{1}$ leaves the protocol, we have

$$
\mathcal{F}_{C_{1}}^{L C}=\frac{2}{3}\{1-q(1-q)\}+\frac{4}{3} \alpha(1-\alpha)\{1-2 q(1-q)\}
$$

Note that if the knowledge of which node $\left(C_{1}\right.$ or $\left.C_{2}\right)$ suffers from more noise is available, the DC protocol can be chosen such that the highest fidelity obtained is

$$
\mathcal{F}^{D C}=\max \left\{\mathcal{F}_{C_{1}}^{L C}, \mathcal{F}_{C_{2}}^{L C}\right\} .
$$

Therefore, we can assert that the fidelity obtained in the TD-TC protocol is equal or greater than the linear chain scheme. Here, we have assumed that the party (if any) which leaves the protocol, is typically unknown.

\subsection{Generalized W state: Quantitative analysis}

We now consider the similar scenario as discussed above, when the initial state is the gW state. Fidelities in two different processes under different noisy channels are listed in Table 5. Note that in case of TD-TC and local measurement-based schemes, both the bit flip and the bit-phase flip errors have similar effects on fidelities. In both the cases, the local measurement-based single-path protocol outperforms the two-path ones for most of the regions in system parameters of $\mathrm{gW}$, i.e., in $(\alpha, \beta)$-plane. On the other hand, there is a contrasting situation in presence of phase damping and phase flip errors - TD-TC yields a better fidelity than the single-path case for almost the entire parameter space of $\mathrm{gW}$ state. Moreover, there exists a finite region in the $(\alpha, \beta)$-plane of the shared $\mathrm{gW}$ state with local amplitude damping noises at $C_{1}$ where multipath performs better than the single ones.

To put things in a quantitative perspective, we calculate percentages of $\mathrm{gW}$ states for which two-path TD-TC protocol provides better quantum capacities than that of the local measurement-based single-path ones under the actions of various local noises. For such comparison, we fix the noise parameter at $p=0.3$, for all the noise models. We generate $10^{6} \mathrm{gW}$ states according to the parameterisations, given in [35]. The observations are the following:

1. For bit flip and bit-phase flip noises, only $9.1 \%$ of the states are useful in TD-TC setting.

2. In case of amplitude damping channel, the percentage of the TD-TC with multipath goes up to $41.9 \%$.

3. For phase damping and phase flip errors, $\mathcal{F}_{2}^{l}(\mathrm{gW}) \leq \mathcal{F}^{D C}(\mathrm{gW})$ holds for $80.3 \%$ and $92.6 \%$ respectively, thereby establishing the two-path protocol as a better ones than the local measurement-based ones. 

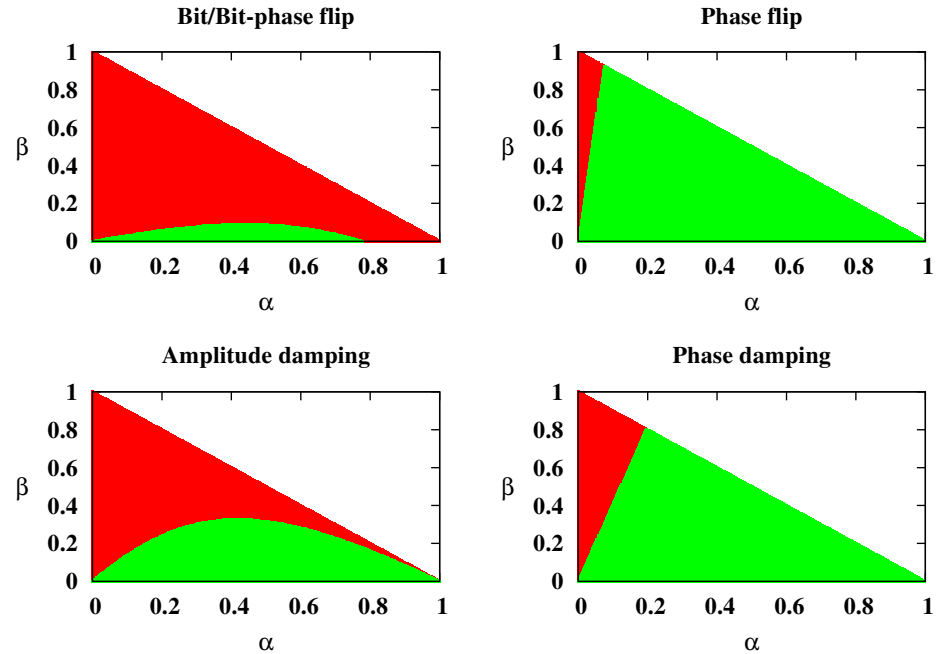

Figure 5: The green regions indicate the $\mathrm{gW}$ state in the $(\alpha, \beta)$-plane. In this region, teleportation via distribution and concentration protocols yields a higher fidelity than the local measurement-based single-path ones which is marked by red. Here $p=0.3$. Both axes are dimensionless.

\section{Multiple blocks of Multipath vs. Local measurement-based Protocols}

We now illustrate the multiple blocks scheme with tripartite states which can be easily generalized to arbitrary number of parties. As shown in Fig. 6, the total length between the sender and the receiver is divided into arbitrary, say $m$, number of units (blocks) - each unit shares two copies of the same given state as in Fig. 1, which execute a multipath TD-TC scheme following the same steps as given in the beginning of Sec. 2. The output state after implementing TD-TC protocol for $i-1$ blocks, becomes the input state for the $i^{\text {th }}$ block. See Fig. 6(a). We are interested in evaluating quantum capacities of an entire quantum channel in terms of the fidelity, $\mathcal{F}_{m}^{D C}$, after $m$ blocks. We will compare the above scenario with the local measurement-based scheme. In the local measurement-based scheme, as before, one of the Claire's of each block, perform optimal local measurements, reducing the entire block structure to a single-path, consisting of $2 m$ bipartite states as depicted in Fig. 6(b).

When three-qubit gGHZ states are used as quantum channels and Bell measurements as well as the same unitary operations are performed as described before, the fidelity for the entire QR process reads as

$$
\begin{aligned}
F_{m}(g G H Z) & =2^{2 m} \alpha^{m}(1-\alpha)^{m} \int 2|a|^{2}|b|^{2} d^{2} a d^{2} b \\
& +\sum_{k=0}^{2 m}\left(\begin{array}{c}
2 m \\
k
\end{array}\right) \alpha^{k}(1-\alpha)^{2 m-k} \int\left(|a|^{4}+|b|^{4}\right) d^{2} a d^{2} b \\
& =\frac{2}{3}+\frac{2^{2 m}}{3}(\alpha(1-\alpha))^{m} \leq \mathcal{F}_{m}^{D C}(g G H Z) .
\end{aligned}
$$

On the other hand, if one obtains $m$ blocks of single-path quantum channels (which therefore consists of $2 m$ bipartite states) by optimal local measurements which in this case is $\{|+\rangle,|-\rangle\}$, iterative methods leads to the fidelity given by

$$
\mathcal{F}_{m}^{l}(g G H Z)=\frac{2}{3}+\frac{2^{2 m}}{3}(\alpha(1-\alpha))^{m},
$$

which happens to coincide with $F_{m}(g G H Z)$, which in turn is upper bounded by $\mathcal{F}_{m}^{D C}(g G H Z)$. If the similar QR problem is considered for the shared generalized $\mathrm{W}$ states of $m$ blocks, the fidelity, after a rather tedious algebraic iterative calculation, is given by

$$
F_{m}(g W)=\frac{2}{3}\left(1+2^{m-1}(2 \alpha+\beta)^{m}(1-\alpha-\beta)^{m}\right)
$$




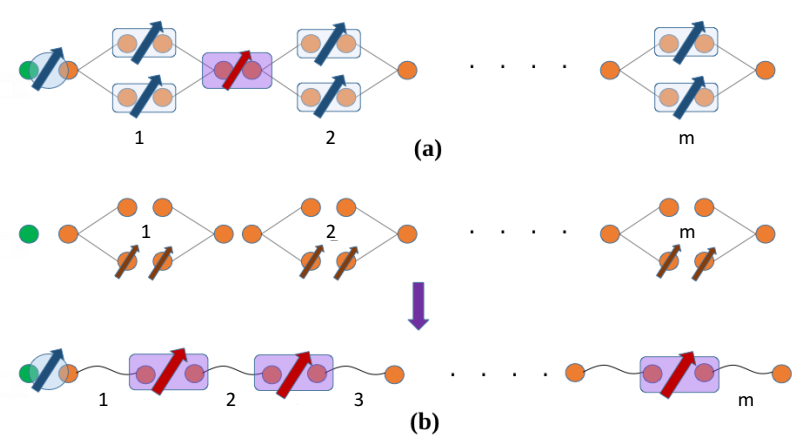

Figure 6: (a) Multipath QR: a schematic diagram of QR with $m$ blocks, each consists of a multipath TD-TC scenario with shared tripartite entangled quantum states. (b) Local measurement-based QR: in each block, one of the parties performs an optimal local measurement on her/his qubits, which converts each block to two single-path quantum channels.
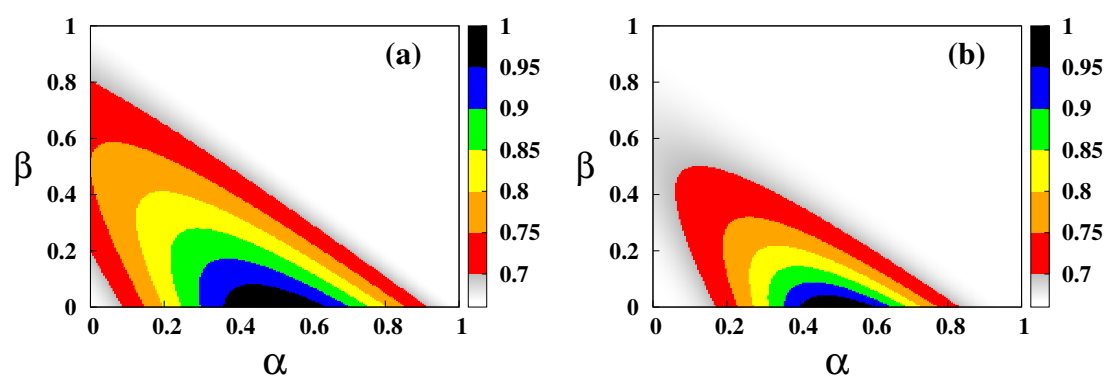

Figure 7: Contour plots of fidelities for multipath TD-TC scheme with few blocks in $(\alpha, \beta)$-plane of the generalized W state. Number of blocks are chosen as (a) $m=2$, (b) $m=4$. Both the axes are dimensionless.

On the other hand, the local measurement-based protocol leads to the fidelity

$$
\mathcal{F}_{m}^{l}(g W)=\frac{2}{3}+\frac{2^{m}}{3} \beta^{m}(1-\alpha-\beta)^{m}
$$

For comparison, $F_{m}(\mathrm{gW})$, for $m=2$ and $m=4$, is depicted in Fig. 7. We observe that the region in the $(\alpha, \beta)$-plane, in which the specific TD-TC protocol shows quantum advantage, shrinks with the increase of the number of blocks. Moreover, comparing Eqs. (37) and (38), we find that the benefit of the two-path scheme over the single-path ones by using $\mathrm{gW}$ states reduces with $m$.

\section{Multiple Claires in a single block of the TD-TC scheme}

Instead of considering multiple distribution-concentration blocks which leads to a repeater like situation, in this section, we consider the effect of multiple Claires (say, $n$ of them) in a single distribution-concentration block. Specifically, if we construct the distribution-concentration block using a $(n+1)$-qubit gGHZ state, which contains $n$ Claires, we find that the obtained teleportation fidelity is independent of $n$. This is because, due to the symmetry of the gGHZ states, the final states with Bob and their corresponding probabilities remain unaltered with changing $n$. In particular, for Bell measurements performed at $n$ Claires, in principle, one expects, $4^{n}$ clicking combinations. However, as also noticed in the two-Claire situation, for gGHZ states, the clicks occur with non-zero probability iff all the outcomes are either from the $\phi$-group or from the $\psi$-group. Therefore, for $n$ Claires, one only needs to consider $2^{n}(\phi$ group $)+2^{n}(\psi$ group $)=2^{n+1}$ outcome combinations. Moreover, the post-measurement states for any $\phi(\psi)$ group 
clickings can be respectively mapped to states that were obtained in the two-Claire scenario (see Table. 1) via local unitary operations, thereby making the fidelity independent of the number of Claires. This further suggests, that the our protocol for the gGHZ states are optimal resulting the best teledistribution and teleconcentration possible for a gGHZ state with a given value of $\alpha$, which inturn measures its genuine multiparty entanglement content [45, 46]. We now want to contrast the two incremental settings considered in the manuscript:

1. Increasing the number of distribution-concentration blocks $(m)$, leading to a repeater like scenario, and

2. Increasing the number of Claires $(n)$ in a distribution-concetration block.

Our investigations show that gGHZ states respond in qualitatively different manner to these increments. In particular, one gets reduction of fidelity on increasing $m$ as shown in Eq. (35), while it is robust to increasing $n$, as argued above. The robustness of fidelity on increasing $n$ implies that in principle, there is no upper bound to the number of intermediate parties (Claires) that one may employ in a given distribution-concentration block. Recall, one of our motivations to introduce multiple Claires was to guarantee that the teleportation protocol gets completed at the stipulated time. If the intermediate party is not trustworthy, she/he might teleport the state before the predecided time. To reduce this possibility, Alice decides to resort to multiple Claires, so that the process is completed if and only if all the mediators complete their actions. Therefore, even if some Claires break the trust, Bob does not receive the state before the predecided time. Let us elaborate this with an example. Suppose the probability that each of the Claires break trust, i.e, completes the protocol before the stipulated time is $p$. Then for $n$ Claires, the probability that the protocol gets completed before the required time is $p^{n}$, which approaches zero for large $n$. Therefore, the correct timing of the protocol can be ensured by involving more Claires as per our wish without hampering the fidelity at all.

In addition the above result right away implies that when one considers a repeater-like scenario, it yields the same fidelity as in Eq. (35) since the output states and their probabilities after a distribution-concentration block remain unchanged for any $n$. Our analysis further reveals that the linear chain obtained by considering $(n-1)$ local measurements, each performed in the $\{|+\rangle,|-\rangle\}$ bases as seen in Eq. (19), results in the same two-party state as obtained in the two-Claire scenario. Therefore, the fidelity for the single-path reduced linear chain setting also remain unaltered, and is given in Eq. (36).

In the noisy case, when only one of the $n$ Claires suffer from bit flip, phase flip, or bit phase flip noise, the fidelities again remain insensitive to $n$. Therefore, features of detection, rectification or both of these noises remain true even when the number of intermediate Claires increases. These computations can be exactly carried out like in the noiseless case, noting that the clicking sectors of bit flip and bit phase flip noises are orthogonal to the noiseless scenario, while phase flip noise share the same clicking sector as the noiseless gGHZ state. Therefore, bit flip and bit phase flip errors are auto-detected. Now one can design the protocol to correct either bit flip or bit phase flip errors. Lastly, we mention that the $n$-Claire-scenario can only be tackled for the gGHZ state and its noisy variants, for which symmetries simplify the calculations.

\section{Conclusion}

Quantum teleportation is a pioneering discovery which forms one of the pillars in the success story of quantum information science. Its exclusivity to the quantum domain puts it in sharp contrast with classical ideas. In this work, we have designed a multiparty teleportation protocol which offers better fidelities compared to single path schemes for some ranges of state parameters both in the noiseless and noisy scenarios. In addition to this, we have also argued that such multiparty schemes (involving distribution and concentration) can be useful in delayed teleportation which necessitates inclusion of multiple paths and intermediate parties. Specifically, we have considered a teleportation protocol in which the teleporter wants the teleportation process to be completed at some later, but fixed time, when she/he would not be available in her/his port. So, the teleportation must be completed by intermediate parties who would complete the process at the predecided time. However, to assure the perfect timing of the protocol, one opts for multiple intermediaries, so that even if some of them attempt a premature completion, the process gets accomplished only when all the intermediate parties complete their actions. We intended to achieve this using two entangled multiparty states, where one is used to "teledistribute" the information, while the other "concentrates" it back at the predecided time. In particular, we have investigated the performance of this multiparty protocol (in terms of its fidelity) for two important families of three-qubit states, the generalized Greenberger-Horne-Zeilinger (gGHZ) and the generalized W states. We have then compared them with a protocol consisting of linear chains obtained from these multiparty states 
by performing optimal local measurements in one of the nodes. In both noiseless and noisy scenarios, we have shown that for certain families of multiparty shared states, one shot capacities, in terms of average output to input fidelity, of multipath protocols are strictly higher than that of the corresponding single-path cases. We have also observed that the protocol proposed in this paper inherently possesses a noise correcting mechanism, when local noise is either bit flip or bit-phase flip, acting on one of the parties of the shared gGHZ state. Moreover, we have found the capacities of long distance quantum channels, consisting of arbitrary number of multipartite as well as bipartite units by using iterative methods. Advantages in quantum state transfer by using multiple path protocol show the importance of creating multipartite entangled states in quantum communication protocols over the bipartite ones.

Acknowledgement: This research was supported in part by the 'INFOSYS scholarship for senior students'.

\section{References}

[1] R. Horodecki, P. Horodecki, M. Horodecki, and K. Horodecki, Rev. Mod. Phys. 81, 865 (2009).

[2] C. H. Bennett and G. Brassard, Proceedings of IEEE International Conference on Computers, Systems and Signal Processing, Bangalore, India (IEEE, NY, 1984).

[3] A. Ekert, Phys. Rev. Lett. 67, 661 (1991); N. Gisin, G. Ribordy, W. Tittel, and H. Zbinden, Rev. Mod. Phys. 74, 145 (2002); V. Scarani, H. Bechmann-Pasquinucci, N. J. Cerf, M. Dušek, N. Lütkenhaus, and M. Peev, Rev. Mod. Phys. 81, 1301 (2009).

[4] C. H. Bennett, and S. J. Wiesner, Phys. Rev. Lett. 69, 2881 (1992); S. Bose, M. B. Plenio, and V. Vedral, J. Mod. Opt. 47, 291 (2000); T. Hiroshima, J. Phys. A: Math. Gen. 34, 6907 (2001); G. Bowen, Phys. Rev. A 63, 022302 (2001); M. Horodecki, P. Horodecki, R. Horodecki, D. Leung, and B. Terhal, Quantum Inf. and Comput. 1, 70 (2001); X. S. Liu, G. L. Long, D.M. Tong, and F. Li, Phys. Rev. A 65, 022304 (2002); M. Ziman and V. Buẑek, ibid. 67, 042321 (2003); D. Bruß, G. M. D’Ariano, M. Lewenstein, C. Macchiavello, A. Sen(De), and U. Sen, Phys. Rev. Lett. 93, 210501 (2004); For a recent review on quantum communication, see e.g. A. Sen(De) and U. Sen, Physics News 40, 17 (2010) (arXiv:1105.2412).

[5] C. H. Bennett, G, Brassard, C. Crépeau, R. Jozsa, A. Peres, and W. K. Wootters, Phys. Rev. Lett. 70, 1895 (1993).

[6] C. H. Bennett, F. Bessette, G. Brassard, L. Salvail, and J. Smolin, J. Cryptology 5, 3 (1992); K. Mattle, H. Weinfurter, P. G. Kwiat and A. Zeilinger, Phys. Rev. Lett. 76, 4656 (1996); D. Leibfried, R. Blatt, C. Monroe, and D. Wineland, Rev. Mod. Phys. 75, 281 (2003); L. M. K. Vandersypen and I. L Chuang, Rev. Mod. Phys. 76, 1037 (2005); H. Hafner, C. F. Roose, and R. Blatt, Phys. Rep. 469, 155 (2008); K. Singer, U. Poschinger, M. Murphy, P. Ivanov, F. Ziesel, T. Calarco, and F. Schmidt-Kaler, Rev. Mod. Phys. 82, 2609 (2010); L.-M. Duan, and C. Monroe, Rev. Mod. Phys. 82, 1209 (2010); and references therein.

[7] J.-W. Pan, Z.-B. Chen, C.-Y. Lu, H. Weinfurter, A. Zeilinger, and M Żukowski, Rev. Mod. Phys. 84, 777 (2012); S. Pirandola, J. Eisert, C. Weedbrook, A. Furusawa, and S. L. Braunstein, Nat. Photonics 9, 641 (2015).

[8] A. R. Calderbank and P. W. Shor, Phys. Rev. A 54, 1098 (1996); A. M. Steane, Phys. Rev. Lett. 77, 793 (1996); A. M. Steane, Phys. Rev. A 54, 4741 (1996); A. R. Calderbank, E. M. Rains, P. W. Shor, and N. J. A. Sloane, Phys. Rev. Lett. 78, 405 (1997).

[9] H. J. Briegel, D. Browne, W. Dür, R. Raussendorf, and M. van den Nest, Nat. Phys. 5, 19 (2009).

[10] H. J. Kimble, Nature 453, 1023 (2008).

[11] M. Beck, Phys. Rev. Lett. 84, 5748 (2000); R. T. Thew, K. Nemoto, A. G. White, and W. J. Munro, Phys. Rev. A 66, 012303 (2002); M. Christandl and R. Renner, Phys. Rev. Lett. 109, 120403 (2012).

[12] V. Giovannetti, S. Lloyd, and L. Maccone, Phys. Rev. Lett. 96, 010401 (2006); V. Giovannetti, S. Lloyd and L. Maccone, Nat. Photonics 5, 222 (2011).

[13] M. Lewenstein, A. Sanpera, V. Ahufinger, B. Damski, A. Sen(De), and U. Sen, Adv. Phys. 56, 243 (2007); L. Amico, R. Fazio, A. Osterloh, and V. Vedral, Rev. Mod. Phys. 80, 517 (2008).

[14] P. Horodecki, M. Horodecki, R. Horodecki, Phys. Rev. A 60, 1888 (1998).

[15] P. Horodecki, M. Horodecki, and R. Horodecki, Phys. Rev. Lett. 82, 1056 (1999).

[16] D. Bouwmeester, J.-W. Pan, K. Mattle, M. Eibl, H. Weinfurter and A. Zeilinger, Nature 390, 575 (1997); D. Boschi, S. Branca, F. De Martini, L. Hardy, and S. Popescu, Phys. Rev. Lett. 80, 1121 (1998); X.-S. Ma, T. Herbst, T. Scheidl, D. Wang, S. Kropatschek, W. Naylor, B. Wittmann, A. Mech, J. Kofler, E. Anisimova, V. Makarov, T. Jennewein, R. Ursin and A. Zeilinger, Nature 489, 269 (2012).

[17] Y.-H. Kim, S. P. Kulik and Y. Shih, Phys. Rev. Lett. 86, 1370 (2001); J. Yin, J.-G. Ren, H. Lu, Y. Cao, H.-L. Yong, Y.-P. Wu, C. Liu, S.-K. Liao, F. Zhou, Y. Jiang, X.-D. Cai, P. Xu, G.-S. Pan, J.-J. Jia, Y.-M. Huang, H. Yin, J.-Y. Wang, Y.-A. Chen, C.-Z. Peng and J.-W. Pan, Nature 488, 185 (2012).

[18] A. Furusawa, J. L. Sørensen, S. L. Braunstein, C. A. Fuchs, H. J. Kimble, E. S. Polzik, Science 282, 706 (1998); W. P. Bowen, et al., Phys. Rev. A 67, 032302 (2003); T. C. Zhang, K. W. Goh, C. W. Chou, P. Lodahl, and H. J. Kimble, Phys. Rev. A 67, 033802 (2003); N. Takei, H. Yonezawa, T. Aoki, and A. Furusawa, Phys. Rev. Lett. 94, 220502 (2005); H. Yonezawa, S. L. Braunstein, and A. Furusawa, Phys. Rev. Lett. 99, 110503 (2007); N. Takei, et al., Phys. Rev. A 72, 042304 (2005); N. Lee, et al., Science 332, 330 (2011); M. Yukawa, H. Benichi, and A. Furusawa, Phys. Rev. A 77, 022314 (2008).

[19] J. Yin et al., Science 356, 1140 (2017); S.-K. Liao et al., Satellite-relayed intercontinental quantum network, arXiv:1801.0441; H. Takenaka, A. Carrasco-Casado, M. Fujiwara, M. Kitamura, M. Sasaki, and M. Toyoshima, Nat Photonics 11, 502 (2017).

[20] M. D. Barrett, J. Chiaverini, T. Schaetz, J. Britton, W. M. Itano, J. D. Jost, E. Knill, C. Langer, D. Leibfried, R. Ozeri and D. J. Wineland, Nature 429, 737 (2004); M. Riebe, H. Häffner, C. F. Roos, W. Hänsel, J. Benhelm, G. P. T. Lancaster, T. W. Körber, C. Becher, F. Schmidt- 
Kaler, D. F. V. James and R. Blatt, Nature 429, 734 (2004); S. Olmschenk, D. N. Matsukevich, P. Maunz, D. Hayes, L. M. Duan, and C. Monroe, Science 323, 486 (2009).

[21] N. Solmeyer, X. Li, and Q. Quraishi, Phys. Rev. A 93, 042301 (2016).

[22] M. A. Nielsen, E. Knill and R. Laflamme, Nature 396, 52 (1998).

[23] L. Steffen, Y. Salathe, M. Oppliger, P. Kurpiers, M. Baur, C. Lang, C. Eichler, G. Puebla-Hellmann, A. Fedorov and A. Wallraff, Nature 500, 319 (2013).

[24] J. Sherson, H. Krauter, R.K. Olsson, B. Julsgaard, K. Hammerer, I. Cirac and E. S. Polzik, Nature 443, 557 (2006).

[25] J. G. Ren, et al., Nature 549, 7670 (2017).

[26] S. Muralidharan, L. Li, J. Kim, N. Lütkenhaus, M. D. Lukin, and L. Jiang, Sci. Rep. 6, 20463 (2016).

[27] M. Murao, M. Plenio, S. Popescu, V. Vedral, and P. L. Knight, Phys. Rev. A 57, 4075, (1998); M. Murao, D. Jonathan, M. B. Plenio, and V. Vedral, Phys. Rev. A 59, 156 (1999); Z. Zhao, A.-N. Zhang, X.-Q. Zhou, Y.-A. Chen, C.-Y. Lu, A. Karlsson, and J.-W. Pan, Phys. Rev. Lett. 95, 030502 (2005); S. Koike, H. Takahashi, H. Yonezawa, N. Takei, S. L. Braunstein, T. Aoki, and A. Furusawa, Phys. Rev. Lett. 96, 060504 (2006); M. Radmark, M. Żukowski, and M. Bourennane, New J. Phys. 11, 103016 (2009); A. Sen(De) and U. Sen, Phys. Rev. A 81, 012308 (2010); J. Lee, S.-W. Ji, J. Park, and H. Nha, Phys. Rev. A 94, 062318 (2016); M. M. Cunha, E. A. Fonseca, and F. Parisio, Non-ideal teleportation of tripartite entanglement: Einstein-Podolsky-Rosen versus Greenberger-Horne-Zeilinger schemes, arXiv:1611:01167.

[28] C. H. Bennett, G. Brassard, S. Popescu, B. Schumacher, J. A. Smolin, and W. K. Wootters, Phys. Rev. Lett. 76, 722 (1996); Erratum Phys. Rev. Lett. 78, 2031 (1997); J.-W. Pan, C. Simon, C. Brukner, A. Zeilinger, Nature 410, 1067 (2001).

[29] M. Żukowski, A. Zeilinger, M. A. Horne, and A. K. Ekert, Phys. Rev. Lett. 71, 4287 (1993); S. Bose, V. Vedral, and P. L. Knight, Phys. Rev. A 60, 194 (1999); P. van Loock and S. L. Braunstein, Phys. Rev. A 61, 010302 (R) (1999). A. Sen(De), U. Sen, and M. Żukowski, Phys. Rev. A 68, 062301 (2003).

[30] H. Briegel, W. Dür, J. I. Cirac, and P. Zoller, Phys. Rev. Lett. 81, 5932 (1998); J. Dias, and T. C. Ralph, Phys. Rev. A 95, 022312 (2017).

[31] A. Acín and J. I. Cirac, and M. Lewenstein, Nat Phys 3, 256-259 (2007); S. Perseguers, J. I. Cirac, A. Acín, M. Lewenstein, and J. Wehr, Phys. Rev. A 77, 022308 (2008); G. J. Lapeyre Jr, J. Wehr, and M. Lewenstein, Phys. Rev. A 79, 042324 (2009); S. Perseguers, D. Cavalcanti, G. J. Lapeyre Jr, M. Lewenstein, and A. Acín Phys. Rev. A 81, 032327 (2010); S. Perseguers, M. Lewenstein, A. Acín, and J. I. Cirac, Nat Phys. 6, 539-543 (2010); G. J. Lapeyre Jr, S. Perseguers, M. Lewenstein, and A. Acín, Quantum Inf. Comput. 12, $502-529$ (2012); S. Perseguers, G. J. Lapeyre Jr, D. Cavalcanti, M. Lewenstein, and A. Acín, Distribution of entanglement in quantum networks, Rep. Prog. Phys. 76, 096001 (2013).

[32] F. Rozpedek, et al., Realistic parameter regimes for a single sequential quantum repeater, arXiv: 1705:00043; F. M. Miatto, M. Epping, and N. Lutkenhaus, Hamiltonians for one-way quantum repeaters, arXiv:1710.03063; T. Holz, H. Kampermann, and D. Bruß, Device-independent Secret Key Rate Analysis for Quantum Repeaters, arXiv:1711.06072; F. Kimiaee Asadi, N. Lauk, S. Wein, N. Sinclair, C. O’Brien, and C. Simon, Quantum repeaters with individual rare-earth ions at telecommunication wavelengths, arXiv:1712.05356; M. Zugenmaier, K. B. Dideriksen, A. S. Sørensen, B. Albrecht, and E. S. Polzik, Long-lived non-classical correlations for scalable quantum repeaters at room temperature, arXiv:1801.03286.

[33] Y. Ogata and M. Murao, Phys. Rev. A 77, 062340 (2008).

[34] M. Murao, M. B. Plenio, and V. Vedral, PRA 61, 032311 (2000).

[35] W. Dür, G. Vidal, and J. I. Cirac, Phys. Rev. A 62, 062314 (2000)

[36] D. M. Greenberger, M. A. Horne, and A. Zeilinger, Kluwer Academic, Dordrecht, The Netherlands, 1989.

[37] J. Preskill, Lecture Notes, available at http://www.theory.caltech.edu/people/preskill/ph219/.

[38] M. A. Nielsen and I. L. Chuang, Quantum Computation and Quantum Information (Cambridge University Press, Cambridge, 2000).

[39] M. Murao and V. Vedral, Phys. Rev. Lett. 86, 352 (2001).

[40] The Bell basis consists of four maximally entangled states, given by $\left|\phi^{ \pm}\right\rangle=\frac{1}{\sqrt{2}}(|00\rangle \pm|11\rangle)$, and $\left|\psi^{ \pm}\right\rangle=\frac{1}{\sqrt{2}}(|01\rangle \pm|10\rangle)$, where $\{|0\rangle,|1\rangle\}$ is the computational basis.

\begin{tabular}{|c|c|c|}
\hline \multicolumn{2}{|c|}{ Measurement Outcomes } & Unitaries \\
\hline Claire 1 & Claire 2 & Bob \\
\hline$\left|\phi^{+}\right\rangle$ & $\left|\phi^{+}\right\rangle$ & $I$ \\
\hline$\left|\phi^{+}\right\rangle$ & $\left|\phi^{-}\right\rangle$ & $\sigma_{z}$ \\
\hline$\left|\phi^{-}\right\rangle$ & $\left|\phi^{+}\right\rangle$ & $\sigma_{z}$ \\
\hline$\left|\phi^{-}\right\rangle$ & $\left|\phi^{-}\right\rangle$ & $I$ \\
\hline$\left|\psi^{+}\right\rangle$ & $\left|\psi^{+}\right\rangle$ & $\sigma_{x}$ \\
\hline$\left|\psi^{+}\right\rangle$ & $\left|\psi^{-}\right\rangle$ & $\sigma_{y}$ \\
\hline$\left|\psi^{-}\right\rangle$ & $\left|\psi^{+}\right\rangle$ & $\sigma_{y}$ \\
\hline$\left|\psi^{-}\right\rangle$ & $\left|\psi^{-}\right\rangle$ & $\sigma_{x}$ \\
\hline
\end{tabular}

Table 6: Table of the unitary rotations performed by Bob corresponding to the possible Bell basis outcomes of the Claires. 
[42] C. H. Bennett, P. W. Shor, J. A. Smolin, and A. V. Thapliyal, Phys. Rev. Lett. 83, 3081 (1999); F. Verstraete, M. Popp, and J. I. Cirac, Phys. Rev. Lett. 92, 027901 (2004); M. Popp, F. Verstraete, M. A. Martín-Delgado, and J. I. Cirac, Phys. Rev. A 71, 042306 (2005); D. Sadhukhan, S. S. Roy, A. K. Pal, D. Rakshit, A. Sen(De), and U. Sen, Phys. Rev. A 95, 022301 (2017).

[43] A. Peres, Quantum Theory: Concepts and Methods (Kluwer, Dordrecht, 1993, pp. 131-133).

[44] J. Preskill, Proc. R. Soc. (Lond.) A 454, 469 (1998); D. Gottesman, and I. Chuang, Nature 402, 390 (1999).

[45] A. Sen(De) and U. Sen, Phys. Rev. A 81, 012308 (2010); arXiv:1002.1253 [quant-ph].

[46] The genuine multiparty entanglement $(\mathcal{G})$ content of a $n$-qubit gGHZ $(\sqrt{\alpha}|00 \ldots 0\rangle+\sqrt{1-\alpha}|11 \ldots 1\rangle)$ state as measured by GGM [45] is computed to be

$$
\mathcal{G}(g G H Z)=\left\{\begin{array}{cc}
\alpha & \alpha \leq \frac{1}{2} \\
1-\alpha & \alpha>\frac{1}{2}
\end{array} .\right.
$$

\section{Appendix A. Fidelity of a configuration of two different linear chains}

Consider now a teleportation protocol with two different linear chains, $\left|\psi_{1}\right\rangle_{A C_{1}}=\sqrt{\alpha_{1}}|00\rangle+\sqrt{1-\alpha_{1}}|11\rangle$, connecting Alice and $C_{1}$ and $\left|\psi_{2}\right\rangle_{C_{1} B}=\sqrt{\alpha_{2}}|00\rangle+\sqrt{1-\alpha_{2}}|11\rangle$, connecting $C_{1}$ and Bob. For such a situation, the optimal teleporation fidelity from Alice to Claire (Claire to Bob) can be obtained by performing Bell measurements and unitary rotations as given in Ref. [5]. To compute the optimal fidelity for two linear chains, we again use Bell measurements and the respective unitaries.

Suppose, $|\psi\rangle$, is the arbitrary state that Alice wants to teleport to Bob. In the first step, Alice performs a joint Bell measurement on the unknown state and on her part of the shared state, followed by a classical communication of measurement results to Claire. Claire then applies local unitary operator on her part, depending on the measurement outcomes that Alice obtained. The teleported state (un-normalized) on Claire's part in the first chain, is given in Table A.7.

\begin{tabular}{|c|c|}
\hline Outcome (Alice's) & Teleported state \\
\hline$\left|\phi^{ \pm}\right\rangle$ & $\frac{1}{\sqrt{2}}\left(a \sqrt{\alpha_{1}}|0\rangle+b \sqrt{1-\alpha_{1}}|1\rangle\right)$ \\
\hline$\left|\psi^{ \pm}\right\rangle$ & $\frac{1}{\sqrt{2}}\left(a \sqrt{1-\alpha_{1}}|0\rangle+b \sqrt{\alpha_{1}}|1\rangle\right)$ \\
\hline
\end{tabular}

Table A.7: Un-normalized teleported state in the Claire's port, after Alice performs Bell measurements and Claire rotates her part with proper unitary operators.

In the second step, Claire teleports each of the quantum states that she obtains (see table. A.8) to Bob by applying the similar protocol as above.

\begin{tabular}{|c|c|}
\hline Outcome (Claire's) & Teleported state \\
\hline \multicolumn{3}{|c|}{ For $\frac{1}{\sqrt{2}}\left(a \sqrt{\alpha_{1}}|0\rangle+b \sqrt{1-\alpha_{1}}|1\rangle\right)$} \\
\hline$\left|\phi^{ \pm}\right\rangle$ & $\frac{1}{2}\left(a \sqrt{\alpha_{1} \alpha_{2}}|0\rangle+b \sqrt{\left(1-\alpha_{1}\right)\left(1-\alpha_{2}\right)}|1\rangle\right)$ \\
\hline$\left|\psi^{ \pm}\right\rangle$ & $\frac{1}{2}\left(a \sqrt{\alpha_{1}\left(1-\alpha_{2}\right)}|0\rangle+b \sqrt{\left(1-\alpha_{1}\right) \alpha_{2}}|1\rangle\right)$ \\
\hline \multicolumn{3}{|c|}{ For $\frac{1}{\sqrt{2}}\left(a \sqrt{1-\alpha_{1}}|0\rangle+b \sqrt{\alpha_{1}}|1\rangle\right)$} \\
\hline$\left|\phi^{ \pm}\right\rangle$ & $\frac{1}{2}\left(a \sqrt{\left(1-\alpha_{1}\right) \alpha_{2}}|0\rangle+b \sqrt{\alpha_{1}\left(1-\alpha_{2}\right)}|1\rangle\right)$ \\
\hline$\left|\psi^{ \pm}\right\rangle$ & $\frac{1}{2}\left(a \sqrt{\left(1-\alpha_{1}\right)\left(1-\alpha_{2}\right)}|0\rangle+b \sqrt{\alpha_{1} \alpha_{2}}|1\rangle\right)$ \\
\hline
\end{tabular}

Table A.8: Un-normalized teleported state in Bob's port, depending on Claire's measurement outcomes.

The repetitive iteration of the above protocol for the two linear chains, leads to the total fidelity, given by 


$$
\begin{aligned}
F= & 4 \int d^{2} a d^{2} b\left[\frac { 1 } { 4 } \left[\left(|a|^{2} \sqrt{\alpha_{1} \alpha_{2}}+|b|^{2} \sqrt{\left(1-\alpha_{1}\right)\left(1-\alpha_{2}\right)}\right)^{2}+\left(|a|^{2} \sqrt{\alpha_{1}\left(1-\alpha_{2}\right)}+|b|^{2} \sqrt{\left(1-\alpha_{1}\right) \alpha_{2}}\right)^{2}\right.\right. \\
& \left.+\left(|a|^{2} \sqrt{\left(1-\alpha_{1}\right) \alpha_{2}}+|b|^{2} \sqrt{\alpha_{1}\left(1-\alpha_{2}\right)}\right)^{2}+\left(|a|^{2} \sqrt{\left(1-\alpha_{1}\right) \alpha_{2}}+|b|^{2} \sqrt{\alpha_{1}\left(1-\alpha_{2}\right)}\right)^{2}\right] \\
= & \frac{2}{3}+\frac{4}{3} \sqrt{\alpha_{1} \alpha_{2}\left(1-\alpha_{1}\right)\left(1-\alpha_{2}\right)} .
\end{aligned}
$$

Note, when $\alpha_{1}=\alpha_{2}=\alpha$, Eq. (A.1) reduces to

$$
F=\frac{2}{3}+\frac{4}{3} \alpha(1-\alpha)
$$

\section{Appendix B. Various noisy channels}

Let us briefly briefly discuss about various kinds of noisy channels $[37,38]$ required in the main text.

Bit flip channel: The bit-flip operation is achieved by applying the Pauli operator $\sigma_{x}$. As the name suggests, it flips the quantum state $|0\rangle$ to $|1\rangle$ and vice-versa with a probability $1-p$ while it keeps the state unchanged with a probability $p,(0<p<1)$. Hence in the presence of bit flip channel, a quantum state $\rho$ is transformed as

$$
\rho \stackrel{\text { bit flip }}{\longrightarrow} p \rho+(1-p) \sigma_{x} \rho \sigma_{x} .
$$

Phase flip channel: The phase flip operation transforms $|1\rangle \rightarrow-|1\rangle$ The transformation in this case reads as

$$
\rho \stackrel{\text { phase flip }}{\longrightarrow} p \rho+(1-p) \sigma_{z} \rho \sigma_{z} .
$$

Bit-phase flip channel: Bit-phase flip channel changes a quantum state as follows:

$$
\rho \stackrel{\text { bit-phase flip }}{\longrightarrow} p \rho+(1-p) \sigma_{y} \rho \sigma_{y} .
$$

Amplitude damping channel: A quantum state, $\rho$, under the action of amplitude damping channel, transforms as

$$
\rho \underset{\text { damping }}{\stackrel{\text { amplitude }}{\longrightarrow}} M_{0} \rho M_{0}^{\dagger}+M_{1} \rho M_{1}^{\dagger},
$$

where $M_{i}, i=0,1$, are the Krauss operators, given by

$$
M_{0}=\left(\begin{array}{cc}
1 & 0 \\
0 & \sqrt{1-p}
\end{array}\right), \quad M_{1}=\left(\begin{array}{cc}
0 & \sqrt{p} \\
0 & 0
\end{array}\right),
$$

Satisfying the condition $M_{0}^{\dagger} M_{0}+M_{1}^{\dagger} M_{1}=I$.

Phase damping channel: The Kraus operator representation of the phase damping channel, when a quantum state $\rho$ is passing through it is given by

$$
\rho \underset{\text { damping }}{\stackrel{\text { phase }}{\longrightarrow}} M_{0} \rho M_{0}^{\dagger}+M_{1} \rho M_{1}^{\dagger}+M_{2} \rho M_{2}^{\dagger} \text {, }
$$

where

$$
M_{0}=\sqrt{1-p}\left(\begin{array}{cc}
1 & 0 \\
0 & 1
\end{array}\right), \quad M_{1}=\sqrt{p}\left(\begin{array}{cc}
1 & 0 \\
0 & 0
\end{array}\right), \quad M_{2}=\sqrt{p}\left(\begin{array}{cc}
0 & 0 \\
0 & 1
\end{array}\right) .
$$




\section{Appendix C. Tables}

\begin{tabular}{|c|c|c|c|c|c|c|c|}
\hline \multicolumn{4}{|c|}{ Alice's measurement outcome $\left|\phi^{ \pm}\right\rangle$} & \multicolumn{4}{|c|}{ Alice's measurement outcome $\left|\psi^{ \pm}\right\rangle$} \\
\hline \multicolumn{2}{|c|}{ Outcomes } & \multirow{2}{*}{$\begin{array}{c}\text { Unitary } \\
\text { B }\end{array}$} & \multirow{2}{*}{$\begin{array}{c}\text { Fidelity } \\
\mid\left.\langle\text { out } \mid \phi\rangle\right|^{2} \times 8\end{array}$} & \multicolumn{2}{|c|}{ Outcomes } & \multirow{2}{*}{$\begin{array}{c}\text { Unitary } \\
\text { B }\end{array}$} & \multirow{2}{*}{$\begin{array}{c}\text { Fidelity } \\
\mid\left.\langle\text { out } \mid \phi\rangle\right|^{2} \times 8\end{array}$} \\
\hline$C_{1}$ & $C_{2}$ & & & $C_{1}$ & $C_{2}$ & & \\
\hline$\left|\phi^{+}\right\rangle$ & $\left|\phi^{+}\right\rangle$ & $I$ & $\left(|a|^{2}(\alpha+\beta)+|b|^{2}(1-\alpha-\beta)\right)^{2}$ & $\left|\phi^{+}\right\rangle$ & $\left|\phi^{+}\right\rangle$ & $\sigma_{x}$ & $\left(|a|^{2}(1-\alpha-\beta)+|b|^{2}(\alpha+\beta)\right)^{2}$ \\
\hline$\left|\phi^{+}\right\rangle$ & $\left|\phi^{-}\right\rangle$ & $\sigma_{z}$ & $\left(|a|^{2}(\alpha-\beta)+|b|^{2}(1-\alpha-\beta)\right)^{2}$ & $\left|\phi^{+}\right\rangle$ & $\left|\phi^{-}\right\rangle$ & $\sigma_{y}$ & $\left(|a|^{2}(1-\alpha-\beta)+|b|^{2}(\alpha-\beta)\right)^{2}$ \\
\hline$\left|\phi^{+}\right\rangle$ & $\left|\psi^{+}\right\rangle$ & $\sigma_{x}$ & $\alpha(1-\alpha-\beta)$ & $\left|\phi^{+}\right\rangle$ & $\left|\psi^{+}\right\rangle$ & $I$ & $\alpha(1-\alpha-\beta)$ \\
\hline$\left|\phi^{+}\right\rangle$ & $\left|\psi^{-}\right\rangle$ & $\sigma_{y}$ & $\alpha(1-\alpha-\beta)$ & $\left|\phi^{+}\right\rangle$ & $\left|\psi^{-}\right\rangle$ & $\sigma_{z}$ & $\alpha(1-\alpha-\beta)$ \\
\hline$\left|\phi^{-}\right\rangle$ & $\left|\phi^{+}\right\rangle$ & $I$ & $\left(|a|^{2}(\alpha-\beta)+|b|^{2}(1-\alpha-\beta)\right)^{2}$ & $\left|\phi^{-}\right\rangle$ & $\left|\phi^{+}\right\rangle$ & $\sigma_{x}$ & $\left(|a|^{2}(1-\alpha-\beta)+|b|^{2}(\alpha-\beta)\right)^{2}$ \\
\hline$\left|\phi^{-}\right\rangle$ & $\left|\phi^{-}\right\rangle$ & $\sigma_{z}$ & $\left(|a|^{2}(\alpha+\beta)+|b|^{2}(1-\alpha-\beta)\right)^{2}$ & $\left|\phi^{-}\right\rangle$ & $\left|\phi^{-}\right\rangle$ & $I$ & $\left(|a|^{2}(1-\alpha-\beta)+|b|^{2}(\alpha+\beta)\right)^{2}$ \\
\hline$\left|\phi^{-}\right\rangle$ & $\left|\psi^{+}\right\rangle$ & $\sigma_{x}$ & $\alpha(1-\alpha-\beta)$ & $\left|\phi^{-}\right\rangle$ & $\left|\psi^{+}\right\rangle$ & $I$ & $\alpha(1-\alpha-\beta)$ \\
\hline$\left|\phi^{-}\right\rangle$ & $\left|\psi^{-}\right\rangle$ & $\sigma_{y}$ & $\alpha(1-\alpha-\beta)$ & $\left|\phi^{-}\right\rangle$ & $\left|\psi^{-}\right\rangle$ & $\sigma_{z}$ & $\alpha(1-\alpha-\beta)$ \\
\hline $\mid \overline{\left|\psi^{+}\right\rangle}$ & $\overline{\left|\phi^{+}\right\rangle}$ & $\overline{\sigma_{x}}$ & $\beta(1-\alpha-\beta)$ & $\left|\psi^{+}\right\rangle$ & $\overline{\left|\phi^{+}\right\rangle}$ & $I$ & $\beta(1-\alpha-\beta)$ \\
\hline$\left|\psi^{+}\right\rangle$ & $\left|\phi^{-}\right\rangle$ & $\sigma_{x}$ & $\beta(1-\alpha-\beta)$ & $\left|\psi^{+}\right\rangle$ & $\left|\phi^{-}\right\rangle$ & $I$ & $\beta(1-\alpha-\beta)$ \\
\hline$\left|\psi^{+}\right\rangle$ & $\left|\psi^{+}\right\rangle$ & $I$ & $4 \alpha \beta|a|^{4}$ & $\left|\psi^{+}\right\rangle$ & $\left|\psi^{+}\right\rangle$ & $\sigma_{x}$ & $4 \alpha \beta|b|^{4}$ \\
\hline $\mid \overline{\left|\psi^{+}\right\rangle}$ & $\left|\psi^{-}\right\rangle$ & $I$ & 0 & $\left|\psi^{+}\right\rangle$ & $\mid \overline{\left|\psi^{-}\right\rangle}$ & $\sigma_{x}$ & 0 \\
\hline $\mid \overline{\left.\psi^{-}\right\rangle}$ & $\left|\phi^{+}\right\rangle$ & $\overline{\sigma_{y}}$ & $\beta(1-\alpha-\beta)$ & $\left|\psi^{-}\right\rangle$ & $\left|\phi^{+}\right\rangle$ & $\sigma_{z}$ & $\beta(1-\alpha-\beta)$ \\
\hline$\left|\psi^{-}\right\rangle$ & $\left|\phi^{-}\right\rangle$ & $\sigma_{y}$ & $\beta(1-\alpha-\beta)$ & $\left|\psi^{-}\right\rangle$ & $\left|\phi^{+}\right\rangle$ & $\sigma_{z}$ & $\beta(1-\alpha-\beta)$ \\
\hline $\mid \overline{\left.\psi^{-}\right\rangle}$ & $\left|\psi^{+}\right\rangle$ & $I$ & 0 & $\left|\psi^{-}\right\rangle$ & $\mid \overline{\left|\psi^{+}\right\rangle}$ & $\sigma_{x}$ & 0 \\
\hline$\left|\psi^{-}\right\rangle$ & $\left|\psi^{-}\right\rangle$ & $I$ & $4 \alpha \beta|a|^{4}$ & $\left|\psi^{-}\right\rangle$ & $\left|\psi^{-}\right\rangle$ & $\sigma_{x}$ & $4 \alpha \beta|b|^{4}$ \\
\hline
\end{tabular}

Table C.9: When the initial state is the generalized W states, the computation of fidelity for each measurement outcomes obtained in the ports of Alice, $C_{1}$ and $C_{2}$ are listed. The corresponding unitary operators at Bob's node are also mentioned.

We notice from the table that like the gGHZ states, when $\left|\psi^{+}\right\rangle$clicks in one of the Claire's port, say, $C_{1}, C_{2}$ can never obtain $\left|\psi^{-}\right\rangle$as her measurement outcome. Note, however that the correlations in the measurement outcomes for $\mathrm{gGHZ}$ is more prominent than that of the $\mathrm{gW}$ states. 